\title{
Cyto- and Chemoarchitecture of the Hypothalamic Paraventricular Nucleus in the C57BL/6J Male Mouse: A Study of Immunostaining and Multiple Fluorescent Tract Tracing
}

\author{
Jonathan Biag ${ }^{1}$, Yi Huang ${ }^{1}$, Lin Gou ${ }^{1}$, Houri Hintiryan ${ }^{1}$, Asal Askarinam ${ }^{1}$, Joel D. Hahn², \\ Arthur W. Toga ${ }^{1}$, and Hong-Wei Dong ${ }^{1,3},{ }^{*}$ \\ ${ }^{1}$ Laboratory of Neuroimaging, Department of Neurology, David Geffen School of Medicine at \\ UCLA, Los Angeles, California 90095-7334 \\ ${ }^{2}$ Brain Architecture Center, University of Southern California, Los Angeles, California 90089-2520 \\ ${ }^{3}$ Brain Research Institute, David Geffen School of Medicine at UCLA, Los Angeles, California \\ 90095-7334
}

\begin{abstract}
The paraventricular nucleus of the hypothalamus $(\mathrm{PVH})$ plays a critical role in the regulation of autonomic, neuroendocrine, and behavioral activities. This understanding has come from extensive characterization of the PVH in rats, and for this mammalian species we now have a robust model of basic PVH neuroanatomy and function. However, in mice, whose use as a model research animal has burgeoned with the increasing sophistication of tools for genetic manipulation, a comparable level of PVH characterization has not been achieved. To address this, we employed a variety of fluorescent tract tracing and immunostaining techniques in several different combinations to determine the neuronal connections and cyto- and chemoarchitecture of the PVH in the commonly used C57BL/6J male mouse. Our findings reveal a distinct organization in the mouse PVH that is substantially different from the PVH of male rats. The differences are particularly evident with respect to the spatial relations of two principal neuroendocrine divisions (magnocellular and parvicellular) and three descending preautonomic populations in the $\mathrm{PVH}$. We discuss these data in relation to what is known about PVH function and provide the work as a resource for further studies of the neuronal architecture and function of the mouse PVH.
\end{abstract}

\section{INDEXING TERMS}

neuroendocrine; autonomic; stress; feeding; neral tract tracing

\begin{abstract}
Over the past two decades, the laboratory mouse (Mus musculus) has become the primary mammalian animal model for biomedical research. Principal reasons for this include the availability of its full genomic sequence together with the development of mouse-specific genetic manipulation techniques (such as gene-targeted muta-genesis) that have allowed the
\end{abstract}

\footnotetext{
(C) 2011 Wiley Periodicals, Inc.

"CORRESPONDENCE TO: Hong-Wei Dong, David Geffen School of Medicine at UCLA, 635 Charles E. Young Drive South, Suite 225, Los Angeles, CA 90095-7334. hongwei.dong@loni.ucla.edu.
} 
engineering of mouse models for a broad array of human diseases. In particular, much effort has been devoted to producing and studying mouse models of devastating nervous system diseases (including psychiatric disorders). In 2004, the National Institutes of Health (NIH) initiated the Knockout Mouse Project (KOMP), which aims to produce and phenotype knockouts for all mouse genes (see NIH KOMP website: http://www.nih.gov/science/ models/mouse/index.html; see also Austin et al., 2004). In support of this effort, more than 20,000 genes have been mapped systematically throughout the mouse brain using largescale in situ hybridization (Allen Brain Atlas [ABA], www.brainmap.org; see Lein et al., 2007) and the bacterial artificial chromosome (BAC) transgenic methods (www.gensat.org; see Gong et al., 2003). In addition, several genetic tracing techniques have been developed and applied to study and manipulate cell type-specific neuronal circuits and their underlying functions and behaviors (see, e.g., Choi et al., 2005; Luo et al., 2008). More recently, it was proposed to create a comprehensive connectome (connection matrix) of the mouse brain by combining data from classical neuronal tract tracing, genetic labeling, and other more recent techniques, such as viral vector tracing, Brainbow mice, and array tomography (Livet et al., 2007; Lichtman and Sanes, 2008; Lichtman et al., 2008; Bohland et al., 2009; Micheva et al., 2010). However, despite these efforts, high-spatial resolution studies of mouse brain architecture and neuronal connectivity are relatively few in comparison with the numerous studies that have been carried out for another rodent species, the rat. The disparity in knowledge between rat and mouse is very evident with respect to neuronal connectivity data: Thousands of central neuronal pathways and connections have been identified and described over the past 30 years in the rat (see Bota et al., 2003). In addition, little is known about the degree to which rats and mice differ with respect to the structural and functional organization of the central nervous system (CNS). Understanding these differences will bridge the gap between the existing wealth of data collected in rats and the ongoing genetic and genomic studies in mice. Working toward this goal, some recent studies have sought systematically to examine and/or refine the neuroanatomy of the C57BL/6J mouse. One approach is to use robust gene markers selected from the genomic-wide digital gene expression library of the ABA to refine anatomic delineations previously defined based on Nissl-stained histological preparations (Dong, 2007; Ng et al., 2009; Dong et al., 2009; Fanselow and Dong, 2010). Another approach is to examine neuronal pathways and circuits in different strains of wild-type and transgenic mice using either traditional tract tracing method (see, e.g., Wang and Burkhalter, 2007; Hashimoto et al., 2009; Qin and Luo, 2009) or genetically modified virus tracing (see, e.g., Campbell and Herbison, 2007; Wickersham et al., 2007; Atasoy et al., 2008; Braz et al., 2009; Haubensak et al., 2010). Our approach in this study involved the combined application of classical tract-tracing and fluorescent immunocytochemical techniques to characterize the neuronal connections and cyto- and chemoarchitecture of the hypothalamic paraventricular nucleus (PVH) in young adult C57BL/6J male mice.

The PVH plays a critical role in integrating neuroendocrine, autonomic, and somatic motor activities to maintain homeostasis in response to internal and external environmental challenges (for reviews see Swanson and Sawchenko, 1980; Swanson, 1987; Herman et al., 2003; Watts, 2005). Structurally, the PVH is one of the most complex, heterogeneously organized nuclei of the brain. In the rat, neurons in the PVH fall into three major categories: 
1) the magnocellular neuroendocrine neurons (PVHm) that send their axons directly to the posterior lobe of the pituitary and secrete either vasopressin (VAS) or oxytocin (OXY) into the general circulation; 2) the parvicellular neuroendocrine neurons (PVHp) that synthesize and secrete a variety of releasing or release-inhibiting hormones, such as corticotrophinreleasing hormone (CRH), thyrotropin-releasing hormone (TRH), and somatostatin (SS), into the hypophysial portal system for transport to the anterior pituitary lobe to influence the synthesis and release of hormones from endocrine cells; and 3) the descending preautonomic neurons (PVHd) that send projections to the lower brainstem and/or spinal cord and are presumably involved in coordinating both sympathetic and parasympathetic, as well as somatomotor, responses with endocrine activity (Swanson and Kuypers, 1980; Sawchenko and Swanson, 1982; Swanson and Sawchenko, 1983; Swanson, 1986, 1987; Simmons and Swanson, 2009). According to their projection destinations and chemical properties, the rat PVH has been further subdivided into more than 10 distinguishable parts within its three principal divisions (Swanson and Kuypers, 1980; Simmons and Swanson, 2009). Here we describe the parts of the mouse PVH and identify their rat PVH homologues; we also give an account of the substantial differences that exist in the cellular organization of the PVH in these two rodent species, more specifically the differences in the topological relations of the neuronal phenotypes.

\section{MATERIALS AND METHODS}

All experiments were performed in accordance with the NIH Guidelines for the care and use of laboratory animals, and all procedures were approved by the UCLA Chancellor's Animal Research Committee (ARC). In total 60 young adult male C57BL/6J mice (20-25 g, 8 weeks of age) from The Jackson Laboratory (Bar Harbor, ME) were used.

\section{Retrograde tract tracing}

To identify neuroendocrine neurons in the $\mathrm{PVH}, 30$ animals received intravenous injections of $30 \mu \mathrm{l} 2 \%$ fluorogold (FG; Fluorochrome, Denver, $\mathrm{CO}$ ) through the tail vein using an insulin syringe $(27.5 \mathrm{G})$. The absence of an effective blood-brain barrier for the median eminence and posterior pituitary allows FG in the blood stream to reach the axon terminals of neuroendocrine neurons in these regions, where they can be taken up and transported retrogradely to the parent cell bodies in the PVH (see Swanson and Kuypers, 1980). Seven days after the injections, 20 animals further received stereotaxic injections of the axoplasmic transport inhibitor colchicine (Sigma-Aldrich, St. Louis, MO; 20 1l, $4 \mathrm{mg} / \mathrm{ml}$ in KPBS) through a glass micropipette (50-lm tip diameter) into the right lateral cerebral ventricle. These mice were then killed 18-24 hours after the treatments. Colchicine inhibits the anterograde axonal transport of neuropeptides, thereby maximizing their concentration in neuroendocrine cells, which enhances their subsequent immunocytochemical detection.

Another 30 animals were used to examine distributions of descending preautonomic neurons and their spatial topological correlations with neuroendocrine neurons in the PVH. To label preautonomic neurons that send descending projections to innervate preganglionic sympathetic neurons in the spinal cord, 18 mice received FG or another retrograe tracer, fast blue (FB; Polysciences, Warrington, PA). These injections were made into a thoracic level 
of the spinal cord between the $\mathrm{T} 1$ and $\mathrm{T} 2$ vertebrae, which contains the descending paraventriculospinal fibers in the dorsolateral funiculus (dlf) and their terminals in the intermediolateral column of the spinal cord (IML). For surgeries, mice were anesthetized with isoflurane and mounted on a stereotaxic frame (model 1900 Stereotaxic Alignment System; David Kopf Instruments, Tujunga, CA). A midline incision was made from the base of the neck to $1 \mathrm{~cm}$ below the shoulder blades. The fatty pad and scapulae were retracted to expose the spinal column and trapezius muscle. The T1 vertebra was located and identified by using its enlarged bony spine as a landmark. Blunt dissection of the trapezious muscle was performed until the space between the $\mathrm{T} 1$ and $\mathrm{T} 2$ vertebrae was exposed.

Approximately $0.05 \mu \mathrm{FG}$ or a $2.5 \%$ aqueous suspension of FB was injected unilaterally between the T1 and the T2 vertebrae into the spinal cord using a 34-G beveled NanoFil needle (tip O.D. $185 \mu \mathrm{m}$, tip I.D. $85 \mu \mathrm{m}$; World Precision Instruments [WPI], Sarasota, FL; NF34BV-2) attached to a Pneumatic PicoPump (WPI; SYS-PV820). The needle was held in place for 1 minute after completion of injection before removal.

To label the neuronal population in the $\mathrm{PVH}$ that sends descending projections to parasympathetic motor neurons in the dorsal vagal complex (DMX; dorsal motor nucleus of vagus and the adjacent nucleus of the solitary tract), 12 mice mice received a stereotaxically placed pressure injection of 0.03-0.05 $\mu$ l Red Retrobeads (RR; Lumafluor, Naples, FL) or $0.03 \mu \mathrm{l}$ of a $2.5 \%$ aqueous suspension of FB into the DMX (Bregma $=-7.255 \mathrm{~mm}, \mathrm{ML}=$ $0.5 \mathrm{~mm}, \mathrm{DV}=4.25 \mathrm{~mm})$ through a glass micropipette $(50-\mu \mathrm{m}$ tip diameter) attached to the Pneumatic PicoPump.

\section{Immunohistochemistry and multiple fluorescent immunostaining}

After a 7-day postsurgical recovery period, all animals were perfused with $30 \mathrm{ml}$ of $0.9 \%$ saline, followed by $100 \mathrm{ml}$ of $4 \%$ paraformaldehyde in $0.1 \mathrm{M}$ sodium phosphate buffer (PB; $\mathrm{pH}$ 9.4). Brains were dissected and postfixed overnight at $4^{\circ} \mathrm{C}$ in the same fixative containing $20 \%$ sucrose and frozen in OCT. Frozen brains were then cut on a cryostat at 25$\mu \mathrm{m}$ thickness, and four series of sections were collected into 24-well plates. The first series of sections was mounted on slides, and neurons retrogradely labeled with FG, FB, and/or RR were visualized using a fluorescent microscope. The second series of sections was used for thionine-based Nissl staining as described previously (Dong, 2007).

The third and fourth series of sections were used to reveal different neuronal populations in the PVH via either a single immunohistochemical peroxidase method or multiple fluorescent immunostaining, respectively. Sections were incubated with combinations of appropriate primary antisera to FG and/or five different neuroendocrine neuropeptides (Tabel 1), including oxytocin (OXY), vasopressin (VAS), corticotrophin-releasing hormone (CRH), thyrotropin-releasing hormone (TRH), and somatostatin (SS), for $48-72$ hours at $4{ }^{\circ} \mathrm{C}$. The primary antibodies were diluted in PBS with $0.3 \%$ Triton X-100 and appropriate blocking serum ( $2 \%$ normal donkey serum or $2 \%$ normal goat serum; Millipore, Billerica, MA). For single immunohistochemical staining, sections were incubated further in biotinylated $\operatorname{IgG}$ antibodies (1:200; Vector Laboratories, Burlingame, CA) for 2-3 hours and stained by using a standard $\mathrm{ABC}$ immunoperoxidase procedure (Vector Laboratories). For multiple fluorescent immunostaining, sections were incubated with combinations of appropriate 
secondary antibodies conjugated with different Alexa Fluor dyes (1:500; Molecular Probes, Carlsbad, CA; see Table 2) for 24 hours at $4^{\circ} \mathrm{C}$ before they were mounted onto slides and coverslipped with ProLong Gold antifade mounting medium (Molecular Probes).

\section{Antibody characterization}

Details of antibodies used in this study are given in Tables 1 (primary antibodies) and 2 (secondary antibodies). The following descriptions indicate how each primary antibody was characterized. A general basis for characterizing antibodies used in neuroanatomical studies, which served as a reference, was provided by a set of comprehensive guidelines (Saper and Sawchenko, 2003).

For OXY, a mouse monoclonal OXY antibody was used to identify OXY-expressing neurons (clone 4G11, MAB5296; Millipore). The OXY antibody was characterized by the manufacturer using a competitive ELISA assay, and no reactivity with the closely related arginine VAS, vasotocin, or [Asu1,6,Arg8]-VAS was reported. In tests with this antibody a pattern of labeling was obtained in the mouse PVH equivalent to one reported previously with the same antibody (Kublaoui et al., 2008). In addition, this monoclonal antibody was used to detect OXY in the rat PVH (Ng et al., 2006), and the pattern of labeling obtained was equivalent to the pattern obtained in the rat $\mathrm{PVH}$ using a polyclonal OXY antibody (Simmons and Swanson, 2009).

For VAS, a polyclonal anti-VAS antiserum raised in guinea pig to a synthetic peptide (antigen sequence H-Cys-Tyr-Phe-Gln-Asn-Cys-Pro-Arg-Gly-NH2) was used (Peninsula Laboratories, Belmaont, CA; T-5048). Radioimmunoassay showed no cross-reaction with OXY, met-enkephalin, or pituitary adenylate cyclase-activating polypeptide-38 (PACAP-38). In the mouse PVH, the cellular distribution pattern that we obtained was similar to that reported previously with different anti-VAS antiserum (Ison et al., 1993).

Two polyclonal antisera raised in rabbit were used to localize CRH in the mouse. One (C70) was a gift from W. Vale (Salk Institute) and has been extensively used in the rat (Sawchenko et al., 1984a,b; Markakis and Swanson, 1997); the other was obtained from a commercial source (Bachem, Torrance, CA; AB1760). The two antibodies produced identical patterns of labeling in the hypothalamus of colchicine-treated mice in our study, which are consistent with CRH mRNA expression (in situ hybridization) in the PVH of intact C57BL/6J mouse presented in the ABA (Image Series 292; see http://www.brain-map.org). In addition, green fluorescent protein (GFP) under control of the CRH promoter in bacterial artificial chromosome (BAC)-transgenic mice shows the same distribution pattern in the PVH that we obtained with the current antibody (Alon et al., 2009).

To label TRH, the rabbit anti-pre-pro-TRH polycolonal (pp363J) antiserum, a gift from M. Wessendorf (University of Minnesota), was used. Preincubation of this antibody with an excess $(10 \mathrm{mg} / \mathrm{ml})$ of synthetic peptide abolishes immunolabeling in the rat (Carr et al., 1993). In addition, the pattern of labeling that we obtained in the mouse PVH with our antipre-pro-TRH antibody was identical to the labeling pattern shown previously in the mouse PVH with a sheep antiserum against TRH (Kadar et al., 2010). 
For SS, we used a commercial polyclonal antibody (ImmunoStar, Hudson, WI; 20067) raised in rabbit and directely against SS15-28. The supplier of this antibody reported that positive labeling was abolished after preadsorption with SS, SS25, and SS28. In addition, the pattern of PVH labeling obtained with this antibody in colchicine-treated rats was identical to the pattern obtained with a different monoclonal anti-SS1-14 antibody (Markakis and Swanson, 1997). We also used an extensively utilized antibody to amplify the signal for the fluorescent retrograde tracer FG (rabbit anti-FG; Chemicon, Rosemont, IL; AB153; Risold and Swanson, 1997).

\section{Image acquisition, processing, and mapping}

Images were acquired with a Leica TCS-SP or an Olympus Fluoview1000 (FV1000) confocal microscope or an Olympus AX70 fluorescent microscope equipped with a DP72 CCD digital camera. All images used for cell counting were captured with the Olympus FV1000. In brief, each side of the PVH was captured separately with a $\times 20$ objective, which resulted in an image size of $1,024 \times 1,024$ pixels. The optimal sampling z-thickness $(1.26$ $\mu \mathrm{m})$ was determined by the Olympus Fluoview software. We used different combinations of filter sets: Alexa Fluor 405, Alexa Fluor 488, Alexa Fluor 594, Alexa Fluor 647, and Qdot 605 (for FG). Lasers were set to scan sequentially, to avoid the bleed-through of the fluorophores. All microphotographs were exported in TIFF format and processed in ImageJ (http://rsbweb.nih.gov/ij/index.html), in which images can be analyzed in a 4D stack. In addition to the traditional three dimensions of the $\mathrm{x}, \mathrm{y}$, and $\mathrm{z}$ levels, the color channel allows independent alteration of intensity for each fluorophore at each $\mathrm{z}$ level. The background subtraction plugin was used to take out unevenness in the images. Colocalization of different fluorescent tracers and neuropeptides was determined on the images going through the zsteps. The numbers of individual cell types in the PVH were counted manually with the aid of the ImageJ cell counter plugin. Instead of counting all cell bodies that were fluorescently labeled, only cells with clear DAPI-labeled nuclei through the z-stack were counted. This method minimizes potential counting errors resulting from different orientations of cell bodies and/or indiscrimination of incomplete somas with large proximal dendrites.

For schematics of the distribution patterns of each neuronal population, the images were separated into their individual channels, and a maximum projection (Z-projection/Max Intensity) for each channel was created. The topological spatial distributions of every neuronal population in the PVH labeled with different fluorescent tracers or neuropeptide immunostaning were examined and plotted onto cytoarchitectonic drawings of adjacent thioninstained sections. These were then transferred onto a series of standard digital atlas drawings of the C57BL/6J mouse PVH (Allen Reference Atlas [ARA]; Dong, 2007). We employed histological procedures identical to those used to create the ARA, including perfusions, freezing brains, thickness of sections, and Nissl staining procedures (see Dong, 2007), and used animals of the same age and body weight. This allowed us to match cytoarchitechtonically every individual section through the PVH in the present study to the levels of the ARA as closely as possible. Accounting for the combination of cytoarchitectonic and chemoarchitectonic properties of the $\mathrm{PVH}$, we have modified the original parceling of the mouse PVH in the ARA (Dong, 2007). 


\section{RESULTS}

\section{Cytoarchitecture of the mouse PVH}

To date, the cellular organization of the PVH is best characterized in the rat (Armstrong et al., 1980, 1982; Swanson and Kuypers, 1980; van den Pol, 1982; Swanson and Sawchenko, 1983; Swanson, 1986, 1987; Rho and Swanson, 1989; Armstrong, 2004; Simmons and Swanson, 2009). Thus, it is convenient first to describe the cellular organization of the mouse $\mathrm{PVH}$ in comparison with that of the rat.

The rat PVH is delineated into 10 distinct parts within three major divisions, magnocellular (PVHm), parvicellular (PVHp), and descending (PVHd) divisions, a consensus arrived at through a combination of neural tract tracing, immunocytochemical identification of neuropeptides, and Nissl-based histology (Swanson and Kuypers, 1980; Swanson, 2004; Simmons and Swanson, 2009). Compared with neurons in neighboring PVH divisions, the magnocellular neurons in the rat PVHm are larger, more darkly stained, and more densely packed. They are embedded in, but relatively segregated from, a shell of parvicellular neurons. This rat PVHm cytoarchitecture is strikingly different from that found in mice.

Beginning at the rostral end, the PVH in the rat clearly extends into the preoptic area ventral to the anterior commissure (Simmons and Swanson, 2008, 2009). In contrast, the rostral limit of the mouse PVH is poorly differentiated and difficult to determine in Nissl-stained sections (Fig. 1A). At a midrostral-to-caudal level of the anterior hypothalamic nucleus (AHN; Bregma $=-0.555 \mathrm{~mm}$; level 60 in the ARA, Dong, 2007; Fig. 36 in Franklin and Paxinos, 2008), the mouse PVH begins to appear as a distinct slim cylindrical nucleus (Fig. 1B), and its neuronal packing density is relatively higher than its adjacent hypothalamic differentiations, with the exception of the periventricular part of the PVH (PVHpv), which has a low packing density (Fig. 1A-G). Caudal to this level, the entire mouse PVH, especially its dorsal portion, extends laterally and stands out as a densely packed, darkly stained, triangular nucleus with its medial edge parallel to and its dorsal edge perpendicular to the wall of the third ventricle (Fig. 1C,D). The PVH at this midrostral-caudal level contains both magnocellular and parvicellular neuroendocrine neurons. However, unlike the case in the rat PVH, there is no discernible cytoarchitectural difference between the two neuronal types in the mouse PVH based solely on Nissl staining. At more caudal levels (Fig. 1D,E) of the mouse PVH, the cell packing density and intensity of the Nissl staining decrease in its ventrolateral and dorsomedial regions (immediately lateral to the PVHpv), giving the appearance that these regions are formed by distinct neuronal subpopulations (Fig. 1E,F), which by and large correspond to the ventral medial parvicellular (PVHmpv) and dorsal parvicellular parts (PVHdp) of the rat PVH, respectively (Swanson and Kuypers, 1980; Simmons and Swanson, 2009). At its most caudal level, the mouse PVH forms a lateral wing that extends through the lateral hypothalamic area (LHA) and ends above the descending column of the fornix (Fig. 1G). Most of the neurons at this most caudal level are medium sized and oval or triangular, and their long axes are oriented predominantly in the horizontal plane (Fig. 1G). The neuron packing density and Nissl staining intensity are markedly attenuated in comparison with the more rostral levels. These cytoarchitectural characteristics are comparable to the lateral parvicellular part (PVHlp) and its lateral tip: the 
forniceal part (PVHf) part of the rat $\mathrm{PVH}$, whose neurons have a prominent descending projection.

Overall, the most striking general feature of the mouse PVH, as revealed by its Nissl cytoarchitecture, is a middle region that is clearly more compact than its rostral and caudal parts. Neuronal populations in the caudal mouse PVH, such as the PVHlp and PVHf, can be reliably recognized in Nissl-stained histological sections, although other subdivisions in more rostral levels cannot be as easily differentiated. Consequently, further delineation of the mouse PVH necessitates alternative methods aside from Nissl staining.

\section{General approach to delineating the chemoarchitecture of the PVH}

Our general strategy is illustrated in Figure 2. The entire neuroendocrine population in the hypothalamus was effectively labeled after tail vein injections of FG (Fig. 2A). Multiple fluorescent immunostaining with antisera to $\mathrm{FG}$ and to different neuropeptides was then performed (Fig. 2C). Neurons double labeled with FG and OXY or VAS were identified as two distinct magnocellular neuroendocrine populations (Fig. 2C,a,c,d), and three different cell types of the PVHp were determined by colocalization of FG with three other neuropeptides, CRH, SS, and TRH. To label descending preautonomic neurons in the PVH, different retrograde tracers (FB, RR, or FG) were injected into the spinal cord and/or the DMX (see Fig. 2B). By using this approach, we were able to identify three distinctive descending populations in the mouse PVH that send projections to the DMX, IML, or central gray of the spinal cord (CGS). By using different combinations of multiple fluorescent tract tracing and immunostaining of neuropeptides, we were able to identify all major neuronal phenotypes and directly compare their spatial correlations in the PVH (i.e., Fig. 2C-F), which allowed for further, more accurate delineation of the PVH. We adopted the nomenclature of the PVH proposed by Swanson and his colleagues (Swanson and Kuypers, 1980; Swanson and Sawchenko, 1983; Swanson, 1986, 1987, 2004; Simmons and Swanson, 2009) because of its wide use in numerous anatomic, behavioral, and functional studies.

Figure 3 shows a representative case (T10), which contains different neuroendocrine and preautonomic neuronal populations and illustrates their overall topological spatial relationship within the PVH. The cellular organization of the two major neuroendocrine divisions and three descending preautonomic populations is described in detail in the following sections.

\section{Organization of the magnocellular neuroendocrine division of the PVH (PVHm)}

We identified four distinct parts of the PVHm, the anterior magnocellular (PVHam), medial magnocellular (PVHmm), and posterior magnocellular (PVHpm) parts, the latter of which comprises a medial zone (PVHpmm) and a lateral zone (PVHpml). First, the PVHam was recognized as a small cluster of OXY magnocellular neurons ventromedial to the descending fornix at the level of the medial preoptic area just caudal to the crossing of the anterior commissure (Fig. 4A-D). This group of neurons is included in the anterior commissure nucleus of Armstrong and colleagues (Armstrong et al., 1980, 1982; Armstrong, 2004) and Franklin and Paxinos (2008). Immediately caudal to the PVHam is a cell-sparse gap that 
contains very few neuroendocrine neurons (Fig. 3B). However, at more caudal levels of the $\mathrm{PVH}$, numerous neuroendocrine neurons emerge that appear as a vertically oriented slim cylinder (Figs. 3C, 5A,D; see also Fig. 1B for cytoarchitectural reference of the PVH for the corresponding coronal level). Multiple fluorescent immunostaining revealed that the great majority of neurons at this particular level are magnocellular neuroendocrine neurons, which cluster into two different parts, the dorsally located PVHmm and the ventrally located PVHpmm. The PVHmm is identified here based on two distinctive properties that have been described for its homological counterpart in the rat (Swanson and Kuypers, 1980): 1) it is located between the PVHam and PVHpm, and 2) it contains predominantly OXY neurons, with only a few scattered VAS neurons along the lateral edge of the PVH (Figs. 3C, 5B,E). In Nissl-stained histological preparations, it is difficult to distinguish the PVHmm from its ventrally adjacent PVHpmm (Fig. 1B), but they are readily distinguishable in histological sections with multiple fluorescent immunostaining, because, unlike the PVHmm, the PVHpmm contains both OXY and VAS magnocellular neurons (Figs. 3C, 5C,F).

Immediately caudally, the PVH becomes triangular on Nissl-stained preparation, with its dorsal portion extending laterally (Fig. 1C). The PVHmm disappears, as indicated by dramatically decreased OXY neurons in the dorsal half of the PVH (Fig. 3D). Instead, a group of VAS neurons takes its place and clusters into a small, tightly compacted ball atop the PVH (Figs. 2C, 3D, Fig. 5K), which presumably corresponds to the rat PVHpml of Swanson and Kuypers (1980) or to the lateral magnocellular group (PaLM) of Armstrong and colleagues (Armstrong et al., 1980; Armstrong, 2004). Small numbers of remaining OXY neurons are distributed medially, but very few are dorsal or lateral to the PVHpml (Fig. 3D; also compare Fig. 5H,I with K,L). On the other hand, the PVHpmm, which contains both OXY and VAS neurons, extends into this coronal level and occupies the ventrolateral side of the PVH and is segregated from the newly emerging PVHpml by densely packed parvicellular neurons in the PVHmpd (Figs. 2C, 3D, Fig. 5I,L). Additionally, a considerable number of magnocellular neurons, mostly OXY positive, are scattered in the PVHpv with their bipolar dendrites parallel to the wall of the third ventricle (Figs. 2C, 3D, Fig. 5H,I). This region corresponds to the periventricular part of Armstrong and colleagues in rats (labeled as the PeM; Armstrong et al., 1980, 1982; Armstrong, 2004).

Advancing in the caudal direction, the PVHpml becomes progressively smaller in volume (Fig. 3E; see also Fig. 1D for corresponding cytoarchitectural reference of the PVH at this level), and the parvicellular PVHmpd gradually fills the major portion of the dorsal half of the PVH. The remaining scattered VAS neurons are distributed dorsomedially to the large number of densely packed parvicellular neurons (Fig. 3F). In tandem, on the ventrolateral side of the PVH, the PVHpmm is also gradually replaced by the PVHmpv, which contains almost exclusively descending preautonomic neurons (see the next section). Very few OXY or VAS neuroendocrine neurons remain in the most caudal level of the PVH. However, small numbers of weakly stained OXY and VAS neurons that are much smaller appear in the PVHlp (especially its medial portion). As described for rats (Sawchenko and Swanson, 1982), these are presumably descending projection neurons, in that they are not colocalized with tail vein-injected FG. At this level, abundant OXY- and VAS-positive dendritic arbors extend laterally through the PVHlp toward the LHA. 
Based on our cell count, the mouse PVH contains approximately $1,116( \pm 89 ; n=9)$ OXY neurons and 1,062 VAS neurons ( $\pm 68, \mathrm{n}=10$ ), after applying the Abercrombie factor (1946), which has been used traditionally to avoid double counts in consecutive histological sections. The cell count was performed manually with the aid of ImageJ software based on multiple z-stack photography of a confocal microscope. Once again, only cells with clear DAPI nuclear labeling were counted.

Collectively, these procedures minimize potential errors of double counting. The peak of OXY distribution in the PVH is at the coronal level, which contains both the PVHmm and the PVHpmm (level 60 in ARA; see Figs. 3C, 5B,C), whereas the distribution of VAS neurons reached its peak about $100 \mu \mathrm{m}$ caudally at the level that contains both the PVHpml and the PVHpmm (level 61 in ARA; Figs. 3D, 5K,L).

\section{Organization of the parvicellular neuroendocrine division of the PVH (PVHp)}

The PVHp contains three major neuropeptidergic populations: CRH, SS, and TRH. The topological spatial distributions of these neurons to OXY and VAS in the PVH have been characterized in detail.

CRH parvicellular neuroendocrine neurons-A few CRH neuroendocrine neurons initially appear at the coronal level of the PVHmm, where they are distributed along the lateral edge of the PVH (Fig. 6A,E). A small cluster of CRH neurons also appears in the dorsolateral corner of the PVH (above the PVHmm). Caudally, numerous CRH neurons emerge and form a distinct, densely packed neuronal band in the PVHmpd, which extends dorsolaterally at approximately a $45^{\circ}$ angle from the wall of the third ventricle to the dorsolateral corner of the PVH (Fig. 6B,C; see also Fig. 6J,K for their cytoarchitectural references; these two levels of the PVH are also comparable to Fig. 1C,D). This pattern is consistent with CRH mRNA expression in the PVH of intact C57BL/6J male mice of same age as archived in the ABA online digital gene expression library (Image Series 292 in http://www.brain-map.org). Multiple fluorescent immunostaining revealed that the CRH population in the PVHmpd is virtually ventrolateral to the PVHpml (which contains primarily VAS neurons) and dorsolateral to the PVHpmm (which contains mixed OXY and VAS neurons; Fig. 6F,G). At a more caudal level of the PVH, where the PVHpml and PVHpmm disappear, the remaining CRH neurons form a dense, compact, lens-shaped cluster that sits atop the $\mathrm{PVH}$ and is encompassed by the descending projection neurons in the dorsal parvicellular (PVHdp) and PVHmpv (Fig. 6H; see also Fig. 3F for FG-labeled neurons in the PVHmpd, which are presumably CRH cells). Only very few CRH neurons are distributed in the lateral parvicellular part (PVHlp), although dense CRHimmunoreactive dendritic branches and axons were observed to extend laterally along the PVHlp toward the LHA.

Very few CRH neurons were colocalized with OXY or VAS neurons, although they intermingle on the boundaries of the PVHmpd (Fig. 6E-H). The total number of $\mathrm{CRH}$ neurons in the PVH is about 1,646 $(n=3)$ after the Abercrombie factor is applied. The peak of their distribution is at the coronal level of the PVHpml (see Fig. 6B,C,F,G). 
SS parvicellular neuroendocrine neurons-According to studies of rats (Swanson, 1986, 1987; Simmons and Swanson, 2009), most neuroendocrine SS neurons are distributed in the hypothalamic periventricular zone, including the PVHpv, along the wall of the third ventricle. In the present study, the mouse PVHpv also contains dense FG-labeled neurons, many of which are SS-positive neuroendocrine neurons (Fig. 7A,B). Most of these SS neurons are located medial to OXY and VAS neurons near the third ventricle (7C,D), although some SS neurons are clustered ventral to the PVHpmm (Fig. 7D). Additionally, very few nonneuroendocrine SS-positive neurons are distributed in other parts of the PVH.

In the rat, SS neuroendocrine neurons also exist in the anterior parvicellular part of the PVH (PVHap; Swanson and Kuypers, 1980; Swanson, 1986, 1987), which is located immediately medial to the PVHam, beneath the descending column of the fornix at the rostral hypothalamic level just caudal to the crossing anterior commissure. However, in the mouse brain, the geographically corresponding region (medial to the PVHam and near the descending column of the fornix) is sparsely populated by neuroendocrine cells (see Fig. 4A $-\mathrm{C})$.

TRH parvicellular neuroendocrine neurons: In rats, TRH neurons are more scarce in the PVH compared with CRH and SS neurons (Simmons and Swanson, 2009). In our study, we found many TRH neurons distributed rostral to the PVHmm, which presumably are nonneuroendocrine cells, because the corresponding region does not contains FG-labeled cells. Most of the TRH neuroendocrine neurons are distributed in the PVHmpd, especially in its medial region (adjacent to the PVHpv; Fig. 7E-H). Relatively fewer TRH neurons are distributed in other parts of the PVH, such as in the PVHmm or PVHpmm. The spatial topological relationship of TRH neurons with VAS neurons in the PVHpml is very striking. It appears that quite a few TRH neurons are medial, ventral, and lateral (in the PVHmpd) to the PVHpml, so they virtually surround the densely packed VAS-positive ball (see Fig. 7F). This spatial relationship has not been observed in rats (Simmons and Swanson, 2009).

\section{Organization of three descending preautonomic populations in the PVH}

We characterized three preautonomic neuronal populations in the PVH that send descending projections to the 1) IML, 2) DMX, and 3) CGS of the spinal cord and carefully compared their topological spatial relationship with that of the neuroendocrine populations. The first two neuronal populations and their descending projection pathways to the IML and DMX have been well described previously from rats (Swanson and Kuypers, 1980; Sawhenko and Swanson, 1982; Cechetto and Saper, 1988). However, to our knowledge, the last group of CGS-projecting neurons previously has not been reported.

Descending neurons projecting to the IML-The neuronal morphology and general distribution pattern of neurons in this group are described based on experimental case T11, in which FG was injected into the upper thoracic level of the spinal cord, which receives descending paraventriculospinal fibers from the dlf and their terminals in the IML. In one series of sections through the hypothalamus, FG retrogradely labeled descending neurons were revealed simultaneously with OXY and VAS neurons using combined multiple fluorescent immunostaining (Fig. 8A,C,E,G). Meanwhile, in an adjacent series of sections, 
FG-labeled neurons were revealed with a diaminobenzidine (DAB)-stained immunohistochemical method to show their detailed Golgi-like morphologies (Fig. 8B,D,F,H). Similar distribution patterns of these IML-projecting PVH neurons were observed in different cases with efficient injections of FG or FB in the IML.

Extensive IML-projecting neurons are distributed throughout almost the entire rostral-tocaudal extension of the PVH. Rostrally, at the level of the PVHmm, a few retrogradely labeled neurons are distributed surrounding the magnocellular neurons (Fig. 8A,B). Most of these descending neurons are medium-sized and fusiform or round, with two primary dendrites that run parallel along the lateral or dorsal edges of the $\mathrm{PVH}$, but remain confined within the PVH. A few large triangular neurons with three or more thick primary dendrites also exist along the lateral edge of the PVH (Fig. 8B). Only very few neurons extend their dendrites and axons laterally beyond the PVH into the LHA.

Further descending neurons appear in more caudal levels of the PVH. These are clustered in the dorsolateral corner of the PVHmpv that is immediately ventromedial to the PVHpmm (Fig. 8C,D). At this level, quite a few medium-sized to large, fusiform, bipolar descending neurons also exist in the vicinity of the PVHpv with their thick, long dendrites vertically oriented along the wall of third ventricle (Fig. 8D,D'). Many retrogradely labeled neurons are located in the dorsomedial corner of the PVH (medial to the PVHpml; Fig. 8C - F), which presumably is the homological counterpart of the PVHdp in rats (Swanson and Kuypers, 1980). The neurons in this region are small to medium-sized, with their dendrites forming a contour along the dorsomedial corner of the PVH (Fig. 8C - F). Some of the large, thick dendritic arbors also extend medially across the top of the third ventricle into the contralateral side of the PVH (Fig. 8C-F). More neurons appear in the PVHmpv at more caudal levels, where the PVHpml and PVHmpd become smaller. These descending neurons and their extensive dendritic arbors essentially form a "nest" that surrounds the neuroendocrine cells in the PVHpml, PVHmpd, and PVHpmm (Fig. 8C-F). At these levels, the entire PVH appears to be wrapped virtually by an abundant complex of dendritic arbors originating from these descending neurons.

At the most caudal levels of the PVH, numerous IML-descending projection neurons are distributed in the PVHlp (Fig. 8G,H; see Fig. 1G for cytoarchitectural reference). Most of these neurons are small, round or oval bipolar neurons with long dendrites that extend horizontally toward the LHA (Fig. 8G,H). Toward the lateral end of the PVHlp, small numbers of IML-projecting neurons are also located in the PVHf.

Descending neurons projecting to the DMX-In nine experiments, injections of RR or FB were centered in the dorsal medulla that contains the dorsal vagal nucleus and the adjacent nucleus of the solitary tract. To label neuroendocrine neurons, three of these animals also received FG tail vein injections. In another three cases, FG was injected in the spinal cord, and FB was injected into the DMX. This double injection strategy, combined with multiple fluorescent labeling, allowed us to compare directly the topological spatial distribution patterns between different descending projection populations, in correlation with neuroendocrine neurons (see Figs. 2E,F, 9A-C). In all of these cases, DMX-projecting neurons display similar distribution patterns in the $\mathrm{PVH}$ and in several other forebrain 
regions, including the insular cortical area, central nucleus of the amygdala (CEA), rhomboid nucleus of the bed nuclei of stria terminalis (BSTrh), and LHA, as described previously for rats (Swanson and Kuypers, 1980; Cechetto and Saper, 1988).

Unlike the IML-projecting neurons, very few DMX-projecting neurons are distributed in the rostral ends of the PVH (see, e.g., at the level of the PVHmm). Instead, large numbers of DMX-projecting neurons are concentrated in the PVHmpv (Figs. 2D, 9A-C), where they are ventral and medial to neuroendocrine neurons in the PVHmpd and PVHpmm but lateral to the PVHpv (Fig. 9A-C; see Fig. 9D-F for corresponding cytoarchitectural references). These DMX-projecting neurons in the PVHmpv lay ventral and medial to IML-projecting neurons, with minimal overlap (Figs. 2F, 10A,B). Many of these DMX-descending neurons are also present in the PVHlp (Figs. 9C, 10C-F), where they tend to be located more ventrally than IML-projecting neurons. In addition, we found a small number of double labeled neurons in the PVHlp, especially in the medial junction with the PVHmpv, that send collateral projections to both the DMX and the IML (Fig. 10C,D,F). This colabeling was rarely found in other subdivisions of the PVH. This result was confirmed based on our careful examination of every individual contiguous section through the PVH (Fig. 10). Finally, similarly to the case in rats (Swanson and Kuypers, 1980), these DMX-projecting neurons were rarely present in the PVHdp (see Figs. 2F, 4E-G, 10A-C).

\section{Descending neurons projecting to the CGS (PVHd-CGS)-Two of our FB}

injection sites were "accidentally" confined in the central gray region of the spinal cord (CGS) that surrounds the central canal at the cervical and upper thoracic levels (experimental cases T10 and M15; Fig. 2B). Although these two injection sites extend rostrally as far as the caudal end of the medulla, injected tracers did not encroach onto the DMX. These two injections consistently resulted in substantial retrogradely labeled neurons in the insular cortex, CEA, and BST, none of which was labeled with IML injections (e.g., in experimental case T11 described above), a result previously reported for rats (Swanson and Kuypers, 1980). Therefore, we propose that this group of retrogradely labeled neurons represents an independent, novel group in the forebrain that sends descending projections to the spinal cord.

In the PVH, the distribution pattern of these CGS-projecting neurons is also distinguished from that of IML-projecting neurons. Particularly, the number of CGS-projecting neurons is notably lower in the PVHdp but significantly higher in the PVHmpv (Figs. 2C, 3E-G). Considerable numbers of these CGS-projecting neurons first appear ventromedially to the PVHmpd and PVHpmm but are lateral to the PVHpv (Figs. 2C, 3E). The great majority of these neurons are concentrated in the PVHmpv underneath the most caudal end of the PVHmpd (Fig. 3F,G). In the most caudal levels of the PVH, many of these neurons also appear in the PVHlp and PVHf (Fig. 3H).

\section{Overview of the cellular organization of the mouse PVH}

In summary, by combining multiple tract tracing with fluorescent neuropeptide immunostaining, we have identified all major neuroendocrine and preautonomic neuronal phenotypes in the PVH of C57BL/6J mice based on their projection destinations and 
chemical properties. The topological spatial distributions of these neuronal populations were carefully examined and compared within the cytoarchitecture of the PVH revealed by Nissl staining in adjacent series of sections. These neurons were carefully plotted onto eight standard coronal levels through the PVH of the ARA, a standard brain atlas of C57BL/6J mice (Dong, 2007; Fig. 11; see Fig. 1 for corresponding cytoarchitectural reference levels). It appears that, although different types of neurons intermix at different degrees along their borders, the same phenotypes still tend to cluster together and display distribution patterns distinct from those of other populations.

As shown in Figure 11 and summarized in Figure 12, the vast majority of neuroendocrine neurons are concentrated at the rostral (anterior) two-thirds of the PVH (levels 56-62 of the ARA). The PVHam and PVHmm contain primarily OXY neurons. In contrast, the PVHpml contains almost exclusively VAS neurons, whereas the PVHpmm contains both types of magnocellular neurons. The vast majority of SS parvicellular neuroendocrine neurons are located in the PVHpv near the third ventricle, whereas two other parvicellular populations, CRH and TRH, are concentrated primarily in the PVHmpd.

In contrast, the caudal (posterior) one-third of the PVH (levels 63-66 of the ARA) contains predominantly three preautonomic descending neuronal populations that control sympathetic and parasympathetic outflow. These neurons are distributed with a distinct spatial topological pattern in four parts of the PVH. 1) The PVHdp contains primarily IMLprojecting neurons and a small number of CGS-projecting neurons. 2) The PVHmpv contains all three descending neuronal populations, which are topographically arranged in such a manner that IML-projecting neurons are located in the most dorsolateral region adjacent to the PVHpmm, whereas DMX-projecting neurons are distributed more medially, adjacent to the PVHpv and occupy the major portion of the PVHmpv. CGS-projecting neurons tend to be distributed in the middle of the PVHmpv but are largely intermingled with DMX-projecting neurons. This topological spatial relationship has not been reported previously for rats (Swanson and Kuyper, 1980). 3) All three descending neuronal populations exist in the PVHlp, where IML-projecting neurons tend to be distributed more dorsally than the DMX-projecting neurons. 4) The PVHf appears to contain primarily spinal cord-projecting neurons.

\section{DISCUSSION}

A rudimentary description of mouse $\mathrm{PVH}$ cytoarchitecture was provided by Cajal more than a century ago (Cajal, 1904); subsequently, the PVH was described by several researchers to varying levels of detail in several different species over the course of the Twentieth Century (Gurdjian, 1927; Le Gros Clark, 1938; Peterson, 1966). However, despite its early identification in the mouse, it is in rats that the most extensive studies of PVH cellular structure (its cyto- and chemoarchitecture) have been carried out, especially during the preceding 3 decades (Armstrong et al., 1980, 1982; Swanson and Kuypers, 1980; Swanson and Sawchenko, 1980; Rhodes et al., 1981; van den Pol, 1982; Swanson, 1987; Markakis and Swanson, 1997; Armstrong, 2004; Simmons and Swanson, 2008, 2009). With the current study we aimed to provide a description of the cyto- and chemoarchitecture of the 
$\mathrm{PVH}$ in male C57BL/6J mice comparable in its level of detail to the descriptions we have of the rat $\mathrm{PVH}$.

In early mouse brain atlases, the PVH as a structure was recognized, but with little differentiation of its constituent parts (Le Gros Clark, 1938; Sidman, 1971). The first detailed cytoarchitectonic description of the mouse hypothalamus, including the $\mathrm{PVH}$, was reported some 3 decades ago by Broadwell and Bleier (1976), who described the PVH in the mouse brain as "a collection of densely grouped, darkly stained cells of varying size lying in the anterior half of the dorsal region adjacent to the periventricular zone and ependymal lining of the third ventricle." This simple description of the mouse PVH (not much different from Cajal's initial description) indicates a basic topology similar to but an outline shape different from the rat PVH. Broadwell and Bleier (1976) went on to propose a tentative schema for subdividing the mouse PVH into ventromedial and dorsolateral divisions based on histological criteria (such as cell size and packing density), but they did not clearly define the boundaries between their proposed divisions. On the basis of our observations, we suggest that Broadwell and Bleir's "dorsolateral division" may include the PVHm, PVHp, PVHdp, and PVHlp as defined here, whereas their "ventromedial division" may include primarily our PVHmpv. In two recently published brain atlases of the C57BL/6J male mouse (Dong, 2007; Franklin and Paxinos, 2008), the PVH was parceled in greater detail, following the nomenclatures used in two rat brain atlases (Paxinos and Watson, 1997; Swanson, 2004). However, the proposed compartmentalization of the PVH in the present study differs somewhat from the schema described in other mouse brain atlases. For example, the regions defined as the medial and lateral magnocellular parts labeled as PaMM and PaLM in Franklin and Paxinos (2008), which should comprise of VAS and OXY, actually contain primarily $\mathrm{CRH}$ parvicellular neurons according to our findings. Also, we found that the region defined as the dorsal cap (PaDC) by Franklin and Paxinos contains mainly VAS and CRH neuroendocrine neurons instead of IML-descending projection neurons. Furthermore, the region defined as the medial parvocellular part (PaMP) by Franklin and Paxinos (2008), which presumably corresponds to our PVHmpd, actually contains primarily somatostatin and not CRH neurons. We have revised the PVH delineations in the ARA (Dong, 2007) based on the current data, particularly the boundaries between the different parts of the magnocellular and parvicellular neuroendocrine populations (i.e., the boundary between the PVHmm and PVHpmm; see Fig. 11).

These discrepancies are due mainly to three factors. 1) Unlike the rat PVH, the mouse PVH is not well differentiated (Swanson and Kuypers, 1980). It is nearly impossible to distinguish the magnocellular and parvicellular neurons in Nissl-stained histological preparations of the mouse brain (Figs. 1, 2). 2) In contrast to the numerous studies of the rat PVH (Sawchenko and Swanson, 1982; Sawchenko et al., 1984a,b; Markakis and Swanson, 1997; KayNishiyama and Watts, 1999; Simmons and Swanson, 2009), few studies have compared the distribution pattern of neuropeptides in the mouse PVH (Castel and Morris, 1988; Shimada and Ishikawa, 1989; Broberger et al., 1999; Kadar et al., 2010), and none has correlated the neuropeptide populations with the overall PVH cytoarchitecture. 3) As demonstrated here, the cellular organization, especially the topology of different neuronal phenotypes, is substantially different from that of rats, and these complex spatial relations are difficult to 
appreciate unless they are directly compared on the same histological sections using multiple fluorescent labeling techniques as has been done in the present study.

The well-characterized rat PVH (Swanson and Kuypers, 1980; Paxinos and Watson, 1997; Swanson, 2004) has provided us with an excellent comparative model for defining the PVH of its rodent species relative the mouse. However, the limits of this approach become apparent as one attempts to define the homological counterparts of the PVH in the mouse brain. Although the term "homology" has different meanings in different contexts, usage of the term indicates a similarity of features, and in neuroanatomical studies the criteria that can be applied to determine the homology for a given group of neurons in two different extant taxa include 1) topological similarity, 2) topographical similarity, 3) similarity of axonal connections, 4) similarity in their relationships to some consistent feature of the two species, 5) similarity of embryological derivation, 6) similarity in the morphological features of individual neurons that form the group, 7) similarity in the neurochemical attributes of the neurons, 8) similarity in the physiological properties of the neurons, and 9) similarity in the behavioral outcomes of neuronal activity (Butler and Hodos, 2005). Although not all of these criteria can be applied in each instance, the more criteria for which data are available, the stronger the support for a hypothesis of historical homology, that is, phyletic continuity. At first glance it does not seem too difficult to identify most individual "homological" nuclei (areas or regions) in the mouse brain based on its topological and topographic relationships with adjacent brain structures and major landmarks (e.g., ventricles, major fibers bundles) with Nissl-stained histology. For example, the PVH as a whole was recognized in Nisslstained histological sections several decades ago (Le Gros Clark, 1938; Sidman, 1971). However, as discussed, it is impossible to identify reliably every subdivision of the mouse PVH solely on the basis of its cytoarchitecture as revealed by the Nissl-stain method.

To go beyond the limits of Nissl stain-based histology, we combined multiple fluorescent tract tracing with immunostaining methods, which allowed us to determine all neuronal phenotypes in the mouse PVH corresponding to their rat counterparts based on combinations of their axonal connections and neurochemical properties (criteria 3 and 7 listed above). The advantage of this approach was that different neuronal phenotypes in the PVH could be labeled by different fluorescent markers and revealed simultaneously on the same histological sections, thus allowing us to compare their spatial topology directly (crtieria 1, $2,4,6$ ). Accordingly, all different parts of the PVH could be accurately delineated in combination with their cytoarchitectonic locations. Advanced confocal microscopy and imaging processing tools also allowed us to estimate the numbers of different neuronal populations more accurately and to determine whether different neuropeptides or tracers were coexpressed in the same neurons. Additionally, retrograde tracers such as fluorogold could be revealed by an immunohistochemical method, which allowed us to examine their morphology in greater depth (e.g., dendritic arbors and spines [criterion 6]), without complex intracellular labeling methods (Rho and Swanson, 1989). All of these approaches enabled us to characterize in detail the complex cellular organization of the mouse PVH and provide a solid structural ground for further characterization of its physiological properties and behavioral outcomes (criteria 8, 9; for example, physiological and behavioral responses of CRH and VAS neurons in response to stress). 


\section{Organization of the mouse PVHm}

The cellular organization of OXY and VAS magnocellular neuroendocrine neurons in the PVHm has been well characterized in rats (Peterson, 1966; Swanson, 1977, 1986, 1987; Armstrong et al., 1980, 1982; Swanson and Kuypers, 1980; Sofroniew et al., 1981; Hou-Yu et al., 1982; Sawchenko et al., 1984b; Ju et al., 1986; Armstrong, 2004; Simmons and Swanson, 2009). OXY and VAS neurons are differentially distributed in four distinct parts of the PVHm. The PVHam and PVHmm, which were identified as two small parts located more rostrally at the junction of the preoptic and anterior hypothalamic areas, contain almost exclusively OXY neurons, whereas the PVHpm contains both magnocellular populations, with more OXY neurons in the medial zone (PVHpmm) but more VAS neurons in the lateral zone (PVHpml; see Swanson and Kuypers, 1980; Swanson, 1986, 1987; Simmons and Swanson, 2009). In the present study, we have identified the counterparts of these four PVHm parts in the C57BL/6J mouse brain. However, the detailed topological spatial relationships between OXY and VAS neurons and their relationships with other neuronal populations in the mouse PVH are substantially different from those in rats. First, the most distinguishing property of the PVHm in the mouse brain lies in the PVHmm. In rats, the PVHmm is a tiny, compact part embedded in the PVHpv near the third ventricle (Swanson and Kuypers, 1980; Armstrong et al., 1980; Simmons and Swanson, 2009). In contrast, the PVHmm in the mouse brain is not confined in the periventricular zone and contains a much larger population of magnocellular neurons. In multiple fluorescent-immunostaining sections, the mouse PVHmm is readily distinguished from its ventrally adjacent PVHpmm, because the former contains predominantly OXY neurons, whereas the latter contains intermixed OXY and VAS neurons. In the study of Armstrong et al. (1982; see also Armstrong, 2004), the homological part of the PVHmm was named the periventricular part (the PeM), which, together with the anterior commissural part (AC; corresponding to the PVHam defined here and previously in rats by Swanson and Kuypers, 1980), is included in the accessory magnocellular neuroscretory neurons. In agreement with Swanson and Kuypers (1980), we consider the PVHmm as a major part of the PVH rather than an "accessory" to magnocellular neuroesecretory neurons. Indeed, the coronal plane containing both the PVHmm and the PVHpmm (level 61 in ARA; Figs. 3B, 5B,C) contains the largest number of OXY neurons in the mouse PVH.

Next, the PVHpml, which contains primarily VAS in both rats (Swanson and Kuypers, 1980) and mice (as shown here), also displays very distinct cellular organizational properties. In rats, the PVHpml is very prominent, in which VAS neurons are densely clustered in the center as a "ball," surrounded by a "ring" composed of dispersed OXY neurons (Sawchenko et al., 1984b; Swanson, 1986, 1987; Simmons and Swanson, 2009). In the mouse brain, although VAS neurons in the PVHpml are also clustered into a tightly packed "ball", its relative size (relative to the overall size of the PVH at same coronal planes) is much smaller. Also, unlike in rats, only small numbers of OXY neurons are distributed medially to the VAS-positive "ball" and do not form a ring surrounding the PVHpml. Finally, in rats, the PVHpmm, which contains twice as many OXY neurons as VAS neurons (Simmons and Swanson, 2009), is located more rostrally than the PVHpml, and these two parts are not present at the same coronal levels. However, in mice, the PVHpmm and PVHpml are present at the same coronal levels for some a distance 
throughout the PVH, along with the extension of the PVHmpd, which essentially sits between these two magnocellular parts. Nevertheless, these two magnocellular parts are not completely segregated. Instead, they are more or less "continued" by quite a few magnocellular neurons (especially VAS neurons) across the PVHmpd.

Several studies using immunohistochemical methods have also examined the neuronal morphology and distributions of OXY and VAS neurons in C57BL/6J mice (Kublaoui et al., 2008), albino house mice (the Oxford University strain; Castel and Morris, 1988), and ICR and mutant polydipsic (STR/N) strains (Ison et al., 1993). Despite different strains being used, the general distribution patterns of these two magnocellular populations in the mouse $\mathrm{PVH}$ are consistent with the present report. Transgenic mice expressing the rat OXY gene also display mouse-like OXY immunoreactivity patterns in the PVH (Young et al., 1990b) rather than the OXY patterns described for rats (Swanson and Kuypers, 1980).

We estimate that the mouse PVH contains approximately 1,000-1,200 OXY neurons and fewer VAS neurons. These numbers are less than those reported for other strains of mice. For example, Kawamoto and Kawashima (1985) estimated 2,100 OXY and 1,600 VAS neurons in 3-month-old C57BLTw mice. Ison et al. (1993) estimated about 1,600 VAS neurons in mutant polydipsic (STR/N) mice and 1,300 VAS neurons in the PVH of ICR mice that were 6-12 months of age. These differences may be the result of variations in the mouse strains, differences in immunohistochemical methods (e.g., different antibodies, thickness of sections, and immunostaining procedures), or differences in the methodologies for cell counting. Our numbers typically appear to be lower than those reported in these other studies, which is consistent with the method we used to count positive neurons. Only cells with clear DAPI-labeled nuclei through the entire z-stack were counted, and the Abercrombie factor (1946) was applied, both of which potentially circumvent the issue of double counts. Compared with rats (Sawchenko and Swanson, 1982; Swanson et al., 1983; Swanson, 1986, 1987), the numbers of OXY neurons in mice as shown here are significantly lower, but the numbers of VAS neurons are comparable. Further studies might be necessary to investigate how the different ratios between OXY and VAS magnocellular neurons in mice and rats contribute to their behavioral differences.

\section{Organization of the PVHp}

The rat PVHp primarily contains three major neuronal phenotypes that express SS, CRH, and TRH (Swanson, 1986, 1987; Markakis and Swanson, 1997; Simmons and Swanson, 2009). Small numbers of parvicellular neuroendocrine cell also synthesize and release growth-releasing hormone (GRH) and dopamine (Simmons and Swanson, 2009).

Our results indicated that most SS neuroendocrine neurons in C57BL/6J mice are distributed in the periventricular zone, including the PVHpv, along the wall of the third ventricle. This result is by and large consistent with several previous reports from several different mouse strains, including garden dormice (Eliomys quercinus L.; Richoux and Dubois, 1980), ddY mice (Shimada and Ishikawa, 1989), and ICR mice (Nurhidayat et al., 1999). Shimada and Ishikawa (1989) claimed that SS neurons were distributed in the periventricular nucleus but not in the PVH of ddY mice. This disagreement was apparently the result of differences in nomenclature and cytoarchitectonic delineations; Shimada and Ishikawa used the rat brain 
atlas of Paxinos and Watson (1982) to describe SS distributions in the mouse brain. Nurhidayat et al. (1999) also noticed sex differentiation of SS neurons in the ICR mouse hypothalamus at different postnatal developmental stages, which warrants confirmation in $\mathrm{C} 57 \mathrm{BL} / 6 \mathrm{~J}$ mice. In rats, SS neuroendocrine neurons are also distributed in the anterior parvicellular part of the PVH (PVHap), which is located immediately medial to the PVHam (Dierickx and Vandesande, 1979; Sawchenko and Swanson, 1982; Swanson, 1986, 1987; Simmons and Swanson, 2009). As we have described here, it appears that the geographically corresponding region of the PVHap in the mouse brain, medial to the PVHam but lateral to the PVHpv at the preoptic levels, just caudal to the crossing anterior commissure in the vicinity of the descending fornix column, contains very few SS neuroendocrine cells. In addition, significant species differences in SS neurons and fibers in several different nonneuroendocrine regions have been observed between mice and rats. For instance, Shimada and Ishikawa (1989) found dense populations of SS-immunoreactive neurons in two nuclei, the anterior hypothalamic and interpeduncularis nuclei, of the mouse, but not in the rat brain. In the same study, using a radioimmunoassay, they also illustrated that the relative SS content in the mouse brain was two to three times higher than that in the rat.

It is well established that CRH plays a critical role in the maintenance of homeostasis in response to various internal and external stress stimuli and is also implicated in numerous neuropsychiatric diseases (Sawchenko and Swanson, 1985; Swanson, 1986, 1987; Herman et al., 2003; Watts, 2005). The distribution of CRH neurons and their processes has been well characterized in colchicinepretreated, adrenalectomized, or noncholchicine-treated but dehydrated rats (Swanson et al., 1983; Sawchenko et al., 1984a,b; Sawchenko and Swanson, 1985; Markakis and Swanson, 1997; Kay-Nishiyama and Watts, 1999; Simmons and Swanson, 2009). Surprisingly, the distribution of CRH has never been described thoroughly in mice, although many mouse models have been used for studying behavioral and hormonal responses to various stressors (see, e.g., Jacobson et al., 2000; Jeong et al., 2000). The present study shows that, similarly to the case in rats, $\mathrm{CRH}$ neuroendocrine neurons in the mouse PVH are concentrated mostly in the PVHmpd (more than $90 \%$ of the total CRH neurons in the PVH; Sawchenko and Swanson, 1985; Swanson, 1986, 1987). However, the spatial topological relationships between $\mathrm{CRH}$ neurons and the surrounding magnocellular neuroendocrine populations are dramatically different. In rats, the PVHmpd is dorsal to the PVHpmm (at more rostral levels) and medial to the PVHpml (more caudally; Sawchenko et al., 1984b; Simmons and Swanson, 2009). In contrast, the PVHmpd in mice is located ventral and lateral to the PVHpml and dorsolateral to the PVHpmm, essentially the reverse topology of the rat PVH (see Figs. 11D,E, 12). Although CRH neurons are intermingled with magnocellular neurons on their borders, we found very few CRH neurons that are indeed colocalized with those magnocellular neurons in either intact or colchicine-pretreated mice. This result is different from that reported for rats by Sawchenko et al. (1984b); they estimated that $40 \%$ of OXY neurons in the PVHam also express CRH. We found almost no coexpression of CRH with VAS in the PVH in colchicine-pretreated mice, which is consistent with previous results in colchicine-pretreated rats (Sawchenko et al., 1984b). It has been reported that adrenalectomy dramatically changes the immunohistochemical staining patterns for CRH and VAS in the rat PVH and results in many colocalizations of 
these two neuropeptides in parvicellular neuroendocrine cells (Tramu et al., 1983; Kiss et al., 1984; Sawchenko et al., 1984a).

Simmons and Swanson (2009) recently mapped in detail the spatial relationships of two magnocellular neuroendocrine and five parvicellular neuroendocrine neurons in the rat $\mathrm{PVH}$, together with nonneuroendocrine neurons expressing the same neuropeptides. They found that the vast majority of CRH- and SS-immunoreactive neurons in the PVH are neuroendocrine, with minimal nonneuroendocrine neurons in overlapping areas. In contrast, about equal numbers of neuroendocrine and non-neuroendocrine TRH neurons are distributed in the PVH, with most of the former in the caudal half of the nucleus and a majority of the latter in the rostral half of the nucleus. In agreement with this result, we have also found many TRH neurons in the rostral level of the mouse PVH, which presumably are nonneuroendocrine neurons because the region does not contain FG-labeled neuroendocrine cells. Similarly to the case in rats (Simmons and Swanson, 2009), the vast majority of TRH neurons in the PVH are distributed in the medial region of the PVHmpd and adjacent to the PVHpv. In a recent article, Kadar et al. (2010) reported that most neuroendocrine TRH neurons were located in the "compact part" of the PVH in C57BL/6J mice, which includes the PVHmpd described here.

\section{Organization of three descending preautonomic populations in the PVH}

Forebrain descending preautonomic neuronal groups have been well characterized in rats by using classic tract-tracing methods (Saper et al., 1976; Swanson, 1977; Swanson and Kuypers, 1980; Sawchenko and Swanson, 1982; Luiten et al., 1985; Cechetto and Saper, 1988; Geerling et al., 2010). Based on these studies, two different populations of descending neurons are differentially distributed in four different parts, PVHdp, PVHmpv, PVHlp, and PVHf. The PVHdp contains primarily IML-projecting neurons that innervate preganglionic sympathetic motor neurons. In contrast, most neurons in the PVHmpv send projections to innervate the parasympathetic motor neurons in the DMX, whereas the PVHlp contains both of these two phenotypes of descending projection neurons. The PVHf seems to contain primarily spinal cord-projecting neurons. We have identified all four homological parts of the PVHd in C57BL/6J mice in the present study.

The most unexpected result is that, in addition to IML-and DMX-projecting neurons, we identified another distinct descending group in the PVH that presumably terminates in the CGS, based on two cases with retrograde tracers "accidentally" injected into a confined region of the spinal cord central gray without contaminating the IML. Interestingly, the general distribution pattern of these CGS-projecting neurons shares more similarities with that of the DMX-projecting neurons rather than IML-projecting neurons (see Fig. 12). Unlike the IML-projecting neurons, more CGS-projecting neurons are presented in the PVHmpv rather than in PVHdp. Additionally, these CGS-descending neurons were also observed in the insular cortical areas, CEA, and BSTrh (unpublished data), all of which send direct projections to the DMX but not to the IML (Saper et al., 1976; Swanson and Kuypers, 1980). Because these two injection sites were far from the DMX, it is unlikely that the CGSprojecting neurons are from the injected tracer encroaching into the DMX. Thus, we believe 
that these neurons represent an independent descending group that generates forebrainspinal cord projections to terminate specifically in the central gray of the spinal cord.

After injections of an anterograde tracer, Phaseolus vulgaris agglutinin (PHAL), into the rat PVH, Luiten et al. (1985) described labeling as "branches in a complex but organized pattern in the intermediolateral column and in area 10 of Rexed just dorsal to the central canal." Thus, it seems very possible that the CGS-projecting neurons also generate collateral projections to the IML and/or DMX, like those neurons in the PVHlp that send collateral projections to both the IML and DMX in mice (the present study) and rats (Swanson and Kuyper, 1980). The vicinity of the central gray of the spinal cord also contains neurophysin I-, OXY-, and VAS-stained fibers, which presumably arise from the PVH neurons (Swanson, 1977; Swanson and McKellar, 1979). Previously, Dong and Swanson (2003) showed that the BSTrh, which also contains retrogradely labeled neurons after tracer injection into the central gray of the spinal cord as shown in the present study (data not shown here), generates terminals in the central gray of upper spinal cord levels.

There are some substantial differences in the cytoarchitecture of PVHd in mice and rats. For example, the PVHdp in rats is well differentiated and distinguishable as a "lens-shaped" group, forming a "dorsomedial cap" of the PVH, and most of its neurons are intermediate in size, with their long axes extending horizontally (Swanson and Kuypers, 1980; Rho and Swanson, 1989). However, in mice, the homological part of the PVHdp is poorly differentiated and displays a different cytoarchitectonic appearance on Nissl-stained histological sections (Fig. 1D-F). It was defined here based on its topological position in the dorsomedial portion of the $\mathrm{PVH}$ and the evidence that it contains predominantly spinal corddescending projection neurons (IML- and CGS-projecting neurons) but almost no DMXprojecting neurons.

In the PVHmpv, neurons of three descending groups are arranged topographically in such a manner that DMX-projecting neurons are concentrated in the ventromedial portion adjacent to the PVHpv, IML-projecting neurons are located in the most dorsolateral corner, and CGSprojecting neurons are more or less concentrated in the middle but largely intermingled with DMX-projecting neurons. Finally, the PVHlp contains all three major descending projection neurons, where it appears that IML-projecting neurons tend to be more dorsal than that of DMX-projecting neurons. None of these topological relationships has been reported previously. It should be noted that there are additional descending neurons in the PVH that project to the rostroventral medulla and to a few other midbrain and brainstem sites (e.g., the periaquaductal gray and parabrachial nucleus; Pyner and Coote, 2000; Stocker et al., 2006; Biancardi et al., 2010; Geerling et al., 2010), which were not mapped in this study. Their topological and topographic patterns in comparison with other groups in the $\mathrm{PVH}$ remain to be investigated.

One striking, and potentially important, phenomenon is that all of these descending neurons (especially IML-projecting neurons) possess abundant dendritic arbors with rich spines, which extend extensively and essentially "enclose" the entire PVH from the outside, which results in the entire neuroendocrine regions being virtually wrapped by these complex dendritic arbors of descending neurons. This phenomenon is substantially different from that 
found in rats. Rho and Swanson (1989) described in detail the neuronal morphologies of these descending neurons in the latter species using intracellular labeling methods with Lucifer yellow. In agreement with our observations in mice, Rho and Swanson described how most of these descending neurons in rats are medium-sized to large, in a stereotypical tripolar arrangement, and give rise to three major dendrites. Unlike the case in the present study, the dendrites of descending neurons in rat PVH do not "wrap" or "enclose" the neuroendocrine parts. Most of their dendrites stay within their cytoarchitectonic borders in the PVHdp or PVHmpv, and only very few extend into the medial rim of the PVHpml, PVHmpd, or PVHpv. A similar observation for rats has also been reported by Armstrong et al. (1980) using the HRP retrograde tract-tracing method. Although its functional significance remains unclear, it is apparent that the extending dendrites of these descending neurons in the mouse PVH possibly serve as the first frontier to process neuronal inputs to the $\mathrm{PVH}$, thus providing an important interface for the coordination of neuroendocrine and autonomic motor actions in response to internal and external environmental stimuli.

In agreement with the data obtained from rats (Sawchenko and Swanson, 1982), we found a small number of OXY- and VAS-positive descending neurons in the PVHlp (especially the medial portion), considerably fewer than rostrally located neuroendocrine magnocellular neurons. Swanson and Kuypers (1980) reported that at least 10-15\% of neurons send collateral projections to both the spinal cord and the vagal complex in rats when using FB and True Blue. However, in our experiments, we found less than 5\% of neurons in the PVH were double labeled after tracers (FG and FB) were injected both into the spinal cord and into the DMX. It remains to be confirmed whether this discrepancy is a result of species differences or differences in the tracers used.

\section{Mice vs. rats}

The historical importance of the rat to scientific research is reflected in the amount of literature that has been dedicated to it, roughly $50 \%$ more than that dedicated to the mouse (Krinke, 2000). Particularly in the field of neuroscience, most of our current knowledge of mammalian neuroanatomy, including neuronal connectivity, has been based on rats over the past 3 decades (for reviews see Dong, 2007; Bota et al., 2003). However, in the last decade, along with rapid development of genetic and genomic technologies, mice have gradually become the primary animal model for studying numerous neurological and neuropsychiatric diseases. This has disadvantaged many investigators, who regard many aspects of behavior and physiology in rats as more relevant to humans and easier to observe than in mice and who wish to trace their observations to underlying genes. As a result, many have been forced to study questions in mice that might have been better pursued in rats.

Nevertheless, the overall value of systematically examining the structural organization and neuronal connectivity of the mouse brain has yet to be fully appreciated, which is mostly a result of the naive belief that the rat and mouse are so similar in morphology and evolutionarily that examining the mouse neuroanatomy would be redundant. In fact, the differences between these two species may be even greater than the difference between human and other primates. The mouse and the rat shared a common ancestor 12-24 million years ago, whereas man and ape made a similar split from a common primate ancestor only 
5-10 million years ago (Gibbs et al., 2004). Rats and mice have different numbers of chromosomes, and their chromosomes look very different from one another (Gibbs et al., 2004). Correspondingly, there are also significant differences between these two species in genetics, reproductive, developmental, and behavioral aspects. Of particular relevance to the present study, collective data suggest that hormonal responses to experimental stimuli in these two species are quite disparate. For example, in rats, repeated (chronic) restraints may result in habituation, in which the hypothalamic-pituitary axis will decrease its response to the same or homotypic stressor but facilitates responses to a novel, or heterotypic, stimulus (Young et al., 1990a; Li and Sawchenko, 1998; Viau, 2002). In contrast, mice receiving 1hour daily repeated restraint for 7 consecutive days do not exhibit habituation to the stressor; instead, they display facilitation, showing a 20-30 times higher plasma corticosterone concentration, a significant increment of adrenal weight, and a reduction of body weight (Tuli et al., 1995). In addition, repeatedly restrained mice show a significant increase of anxiety and depression-like behavior (Jones et al., 1998; Kim and Han, 2006). Therefore, we hope our present study provides a solid structural basis for further investigating how anatomic differences contribute to these behavioral and hormonal differences in the two most frequently used rodent models in neuropsychiatric studies.

In summary, using a combination of fluorescent immunocytochemistry, tract-tracing, and Nissl-based histology, we have identified and described the parts of the mouse PVH and have shown how each of these parts has a homologous counterpart in the rat PVH. Despite this equivalence, we found striking and substantial differences in the topological relations of the parts and in the distribution of different neuronal phenotypes in the different parts. These findings for the mouse $\mathrm{PVH}$ lead us to recognize that a systematic comparative approach could be usefully applied to other mouse brain regions, and with specific regard to the $\mathrm{PVH}$ we suggest that future functional studies of the PVH in rat and mouse use the present work as a reference to correlate their findings more closely with the underlying cellular neuroanatomy. This work is significant for the following two reasons: 1 ) it will bridge the knowledge gap between the overwhelmingly abundant anatomic, physiological, behavioral, and functional data in rats and ongoing genetic and genomic studies in mice, and 2) understanding the structural similarities and differences between these two closely related species will lead us to deeper insights into the evolution of the brain and greatly help the interpretation and analysis of species differences in behavioral, functional, and genetic studies. Nevertheless, it might be important to emphasize here that the differences between these two rodents should not be overlooked. We reiterate the importance of understanding why mice are mice and not small rats, which will ultimately help us understand why humans are not other mammals.

\section{Acknowledgments}

The CRH antibody was kindly provided by Prof. W. Vale (Salk Institute, La Jolla, CA). The TRH antibody was a generous gift from Prof. M. Wessendorf at the University of Minnesota. The authors also thank Dr. Arshad Khan, Dr. Alan G. Watts, and Mrs. Grace Liang-Franco for critical reading of the manuscript.

Grant sponsor: National Institutes of Health; Grant numbers: R21MH083180 (to H.-W.D.), P41RR013642 (to A.W.T.). 


\section{Abbreviations}

\begin{tabular}{|c|c|}
\hline AHN & anterior hypothalamic nucleus \\
\hline AHNc & anterior hypothalamic nucleus, central part \\
\hline AHNp & anterior hypothalamic nucleus, posterior part \\
\hline BST & bed nuclei stria terminalis \\
\hline $\mathbf{C C}$ & central canal, spinal cord/medulla \\
\hline CEA & central nucleus amygdala \\
\hline CGS & central gray, spinal cord \\
\hline DH & dorsal horn, spinal cord \\
\hline Dlf & dorsolateral funiculus \\
\hline DMX & dorsal motor nucleus of the vagus nerve \\
\hline $\mathbf{f x}$ & columns of the fornix \\
\hline LHA & lateral hypothalamic area \\
\hline IML & intermediolateral column, spinal cord \\
\hline ME & median eminence \\
\hline NC & nucleus circularis \\
\hline $\mathbf{P P}$ & posterior pituitary \\
\hline PVa & periventricular hypothalamic nucleus, anterior part \\
\hline PVpo & periventricular hypothalamic nucleus, preoptic part \\
\hline PVH & paraventricular hypothalamic nucleus \\
\hline PVHam & paraventricular hypothalamic nucleus, anterior magnocellular part \\
\hline PVHap & paraventricular hypothalamic nucleus, anterior parvicellular part \\
\hline PVHdp & $\begin{array}{l}\text { paraventricular hypothalamic nucleus, descending division, dorsal } \\
\text { parvicellular part }\end{array}$ \\
\hline PVHf & paraventricular hypothalamic nucleus, descending division, forniceal part \\
\hline PVHIp & $\begin{array}{l}\text { paraventricular hypothalamic nucleus, descending division, lateral } \\
\text { parvicellular part }\end{array}$ \\
\hline PVHm & mparaventricular hypothalamic nucleus, medial magnocellular part \\
\hline PVHmpd & paraventricular hypothalamic nucleus, medial parvicellular part, dorsal zone \\
\hline PVHmpv & paraventricular hypothalamic nucleus, medial parvicellular part, ventral zone \\
\hline PVHpml & $\begin{array}{l}\text { paraventricular hypothalamic nucleus, posterior magnocellular part, lateral } \\
\text { zone }\end{array}$ \\
\hline
\end{tabular}


PVHpmm paraventricular hypothalamic nucleus, posterior magnocellular part, medial zone

PVHpv paraventricular hypothalamic nucleus, periventricular part

SBPV subparaventricular zone

VH ventral horn, spinal cord

V3 third ventricle

ZI zona incerta

\section{LITERATURE CITED}

Abercrombie M. Estimation of nuclear population from microtome sections. Anat Rec. 1946; 94:239247. [PubMed: 21015608]

Alon T, Zhou L, Pe'rez CA, Garfield AS, Friedman JM, Heisler LK. Transgenic mice expressing green fluorescent protein under the control of the corticotropin-releasing hormone promoter. Endocrinology. 2009; 150:5626-5632. [PubMed: 19854866]

Armstrong, WE. Hypothalamic supraoptic and paraventricular nuclei. The rat nervous system. 3rd ed. Paxinos, G., editor. Amsterdam: Elsevier; 2004. 2004. p. 369-388.

Armstrong WE, Warach S, Hatton GI, McNeill TH. Subnuclei in the rat hypothalamic paraventricular nucleus: a cytoarchitectural, horseradish peroxidase and immunocytochemical analysis. Neuroscience. 1980; 5:1931-1958. [PubMed: 7432630]

Armstrong, WE.; McNeill, TH.; Scholer, J. Cytoarchitectural organization of the hypothalamic paraventricular and anterior commissural nuclei: an immunocytochemical study in the rat. In: Baertschi, A.; Dreifuss, J-J., editors. Neuroendocrinology of vasopressin, corticoliberin and opiomelanocortins. London: Academic Press. p; 1982. p. 33-50.

Atasoy D, Aponte Y, Su HH, Sternson SM. A FLEX switch targets channelrhodopsin-2 to multiple cell types for imaging and long-range circuit mapping. J Neurosci. 2008; 28:7025-7030. [PubMed: 18614669]

Austin CP, Battey JF, Bradley A, Bucan M, Capecchi M, Collins FS. The knockout mouse project. Nat Genet. 2004; 36:921-924. [PubMed: 15340423]

Biancardi VC, Campos RR, Stern JE. Altered balance of gamma-aminobutyric acidergic and glutamatergic afferent inputs in rostral ventrolateral medulla-projecting neurons in the paraventricular nucleus of the hypothalamus of renovascular hypertensive rats. J Comp Neurol. 2010; 518:567-585. [PubMed: 20034060]

Bohland JW, Wu C, Barbas H, Bokil H, Bota M, Breiter HC, Cline HT, Doyle JC, Freed PJ, Greenspan RJ, et al. A proposal for a coordinated effort for the determination of brainwide neuroanatomical connectivity in model organisms at a mesoscopic scale. PLoS Comput Biol. 2009; 5:e1000334. [PubMed: 19325892]

Bota M, Dong HW, Swanson LW. From gene networks to brain networks. Nat Neurosci. 2003; 6:795799. [PubMed: 12886225]

Braz JM, Enquist LW, Basbaum AI. Inputs to serotonergic neurons revealed by conditional viral transneuronal tracing. J Comp Neurol. 2009; 514:145-160. [PubMed: 19274668]

Broadwell RD, Bleier R. A cytoarchitectonic atlas of the mouse hypothalamus. J Comp Neurol. 1976; 167:315-339. [PubMed: 818133]

Broberger C, Visser TJ, Kuhar MJ, Hokfelt T. Neuropeptide Y innervation and neuropeptide-Y-Y1receptor-expressing neurons in the paraventricular hypothalamic nucleus of the mouse. Neuroendocrinology. 1999; 70:295-305. [PubMed: 10567855]

Butler, AB.; Hodos, W. Comparative vertebrate neuroanatomy: evolution and adaptation. Hoboken, NJ: John Wiley \& Sons; 2005. 
Cajal, SR. Textura del Sistema Nervioso del Hombre y de los Vertebrados. Madrid: Moya; 1904. p. 611-1209.

Campbell RE, Herbison AE. Definition of brainstem afferents to gonadotropin-releasing hormone neurons in the mouse using conditional viral tract tracing. Endocrinology. 2007; 148:5884-5890. [PubMed: 17823269]

Carr FE, Reid AH, Wessendorf MW. A cryptic peptide from the preprothyrotropin-releasing hormone precursor stimulates thyrotropin gene expression. Endocrinology. 1993; 133:809-814. [PubMed: 8344217]

Castel M, Morris JF. The neurophysin-containing innervation of the forebrain of the mouse. Neuroscience. 1988; 24:937-966. [PubMed: 3380308]

Cechetto DF, Saper CB. Neurochemical organization of the hypothalamic projection to the spinal cord in the rat. J Comp Neurol. 1988; 272:579-604. [PubMed: 2901438]

Choi GB, Dong HW, Murphy AJ, Valenzuela DM, Yancopoulos GD, Swanson LW, Anderson DJ. Lhx6 delineates a pathway mediating innate reproductive behaviors from the amygdala to the hypothalamus. Neuron. 2005; 46:647-660. [PubMed: 15944132]

Dierickx K, Vandesande F. Immunocytochemical localization of somatostatin-containing neurons in the rat hypothalamus. Cell Tissue Res. 1979; 201:349-359. [PubMed: 509487]

Dong, HW. Hoboken, NJ: John Wiley \& Sons; 2007. The allen reference atlas: a digital color brain atlas of the C57BL/6J male mouse (book + CD-ROM).

Dong HW, Swanson LW. Projections from the rhomboid nucleus of the bed nuclei of the stria terminalis: implications for cerebral hemisphere regulation of ingestive behaviors. J Comp Neurol. 2003; 463:434-472. [PubMed: 12836178]

Dong HW, Swanson LW, Chen L, Fanselow MS, Toga AW. Genomic-anatomic evidence for distinct functional domains in hippocampal field CA1. Proc Natl Acad Sci U S A. 2009; 106:1179411799. [PubMed: 19561297]

Fanselow MS, Dong HW. Are the dorsal and ventral hippocampus functionally distinct structures? Neuron. 2010; 65:7-19. [PubMed: 20152109]

Franklin, KBJ.; Paxinos, G. The mouse brain in stereotaxic coordinates. Amsterdam: Elsevier; 2008.

Geerling JC, Shin JW, Chimenti PC, Loewy AD. Paraventricular hypothalamic nucleus: axonal projections to the brainstem. J Comp Neurol. 2010; 518:1460-1499. [PubMed: 20187136]

Gibbs RA, Weinstock GM, Metzker ML, Muzny DM, Sodergren EJ, Scherer S, Scott G, Steffen D, Worley KC, et al. Genome sequence of the brown Norway rat yields insights into mammalian evolution. Nature. 2004; 428:493-521. [PubMed: 15057822]

Gong S, Zheng C, Doughty ML, Losos K, Didkovsky N, Schambra UB, Nowak NJ, Joyner A, Leblanc G, Hatten ME, Heintz N. A gene expression atlas of the central nervous system based on bacterial artificial chromosomes. Nature. 2003; 425:917-925. [PubMed: 14586460]

Gurdjian ES. The diencephalon of the albino rat. J Comp Neurol. 1927; 43:1-114.

Hashimoto K, Obata K, Ogawa H. Characterization of parabrachial subnuclei in mice with regard to salt tastants: possible independence of taste relay from visceral processing. Chem Senses. 2009; 34:253-267. [PubMed: 19179538]

Haubensak W, Kunwar PS, Cai H, Ciocchi S, Wall NR, Ponnusamy R, Biag J, Dong HW, Deisseroth K, Callaway EM, Fanselow MS, Lu"thi A, Anderson DJ. Genetic dissection of an amygdala microcircuit that gates conditioned fear. Nature. 2010; 468:270-276. [PubMed: 21068836]

Herman JP, Figueiredo H, Mueller NK, Ulrich-Lai Y, Ostrander MM, Choi DC, Cullinan WE. Central mechanisms of stress integration: hierarchical circuitry controlling hypothalamo-pituitaryadrenocortical responsiveness. Front Neuroendocrinol. 2003; 24:151-180. [PubMed: 14596810]

Hou-Yu A, Ehrlich PH, Valiquette G, Engelhardt DL, Sawyer WH, Nilaver G, Zimmerman EA. A monoclonal antibody to vasopressin: preparation, characterization, and application in immunocytochemistry. J Histochem Cytochem. 1982; 30:1249-1260. [PubMed: 6185560]

Ison A, Yuri K, Ueta Y, Leng G, Koizumi K, Yamashita H, Kawata M. Vasopressin- and oxytocinimmunoreactive hypothalamic neurones of inbred polydipsic mice. Brain Res Bull. 1993; 31:405414. [PubMed: 8490739] 
Jacobson L, Muglia LJ, Weninger SC, Pacak K, Majzoub JA. CRH deficiency impairs but does not block pituitaryadrenal responses to diverse stressors. Neuroendocrinology. 2000; 71:79-87. [PubMed: 10686522]

Jeong KH, Jacobson L, Pacak K, Widmaier EP, Goldstein DS, Majzoub JA. Impaired basal and restraint-induced epinephrine secretion in corticotropin-releasing hormonedeficient mice. Endocrinology. 2000; 141:1142-1150. [PubMed: 10698191]

Jones BC, Sarrieau A, Reed CL, Azar MR, Mormede P. Contribution of sex and genetics to neuroendocrine adaptation to stress in mice. Psychoneuroendocrinology. 1998; 23:505-517. [PubMed: 9802125]

Ju G, Liu S, Tao J. Projections from the hypothalamus and its adjacent areas to the posterior pituitary in the rat. Neuroscience. 1986; 19:803-828. [PubMed: 3796817]

Kadar A, Sanchez E, Wittmann G, Singru PS, Fuzesi T, Marsili A, Larsen PR, Liposits Z, Lechan RM, Fekete C. Distribution of hypophysiotropic thyrotropin-releasing hormone (TRH)-synthesizing neurons in the hypothalamic paraventricular nucleus of the mouse. J Comp Neurol. 2010; 518:3948-3961. [PubMed: 20737594]

Kawamoto K, Kawashima S. Plasticity of vasopressin-and oxytocin-containing fibers in the median eminence in hypophysectomized young and old mice. Brain Res. 1985; 330:189-193. [PubMed: 3886075]

Kay-Nishiyama C, Watts AG. Dehydration modifies somal CRH immunoreactivity in the rat hypothalamus: an immunocytochemical study in the absence of colchicine. Brain Res. 1999; 822:251-255. [PubMed: 10082905]

Kim KS, Han PL. Optimization of chronic stress paradigms using anxiety- and depression-like behavioral parameters. J Neurosci Res. 2006; 83:497-507. [PubMed: 16416425]

Krinke, GJ. History, strains and models. In: Bullock, GR.; Bunton, T., editors. The laboratory rat (Handbook of experimental animals). New York: Academic Press; 2000. p. 3-16.

Kublaoui BM, Gemelli T, Tolson KP, Wang Y, Zinn AR. Oxytocin deficiency mediates hyperphagic obesity of Sim1 haploinsufficient mice. Mol Endocrinol. 2008; 22:1723-1734. [PubMed: 18451093]

Le Gros, Clark WE. The hypothalamus: morphological, functional, clinical and surgical aspects. Edinburgh: Oliver \& Boyd; 1938. Morphological aspects of the hypothalamus; p. 1-68.

Lein ES, Hawrylycz MJ, Ao N, Ayres M, Bensinger A, Bernard A, Boe AF, Boguski MS, Brockway KS, Byrnes EJ, Chen L, et al. Genome-wide atlas of gene expression in the adult mouse brain. Nature. 2007; 445:168-176. [PubMed: 17151600]

Li HY, Sawchenko PE. Hypothalamic effector neurons and extended circuitries activated in "neurogenic" stress: a comparison of footshock effects exerted acutely, chronically, and in animals with controlled glucocorticoid levels. J Comp Neurol. 1998; 393:244-266. [PubMed: 9548700]

Lichtman JW, Sanes JR. Ome sweet ome: what can the genome tell us about the connectome? Curr Opin Neurobiol. 2008; 18:346-353. [PubMed: 18801435]

Lichtman JW, Livet J, Sanes JR. A technicolour approach to the connectome. Nat Rev Neurosci. 2008; 9:417-422. [PubMed: 18446160]

Livet J, Weissman TA, Kang H, Draft RW, Lu J, Bennis RA, Sanes JR, Lichtman JW. Transgenic strategies for combinatorial expression of fluorescent proteins in the nervous system. Nature. 2007; 450:56-62. [PubMed: 17972876]

Luiten PG, ter Horst GJ, Karst H, Steffens AB. The course of paraventricular hypothalamic efferents to autonomic structures in medulla and spinal cord. Brain Res. 1985; 329:374-378. [PubMed: 3978460]

Luo L, Callaway EM, Svoboda K. Genetic dissection of neural circuits. Neuron. 2008; 57:634-660. [PubMed: 18341986]

Markakis EA, Swanson LW. Spatiotemporal patterns of secretomotor neuron generation in the parvicellular neuroendocrine system. Brain Res Brain Res Rev. 1997; 24:255-291. [PubMed: 9385456]

Micheva KD, Busse B, Weiler NC, O'Rourke N, Smith SJ. Single-synapse analysis of a diverse synapse population: proteomic imaging methods and markers. Neuron. 2010; 68:639-653. [PubMed: 21092855] 
Ng L, Bernard A, Lau C, Overly CC, Dong HW, Kuan L, Pathak S, Sunkin SM, Dang C, Bohland JW, Bokil H, Mitra PP, Puelles L, Hohmann J, Anderson DJ, Lein ES, Jones AR, Hawrylycz M. An anatomic gene expression atlas of the adult mouse brain. Nat Neurosci. 2009; 12:356-362. [PubMed: 19219037]

Ng YK, Brailoiu GC, Dun SL, Ling EA, Yang J, Chang JK, Dun NJ. Beacon immunoreactivity in the rat hypothalamus. J Neurosci Res. 2006; 83:1106-1117. [PubMed: 16511859]

Nurhidayat, Tsukamoto Y, Sigit K, Sasaki F. Sex differentiation of growth hormone-releasing hormone and somatostatin neurons in the mouse hypothalamus: an immunohistochemical and morphological study. Brain Res. 1999; 821:309-321. [PubMed: 10064817]

Paxinos, G.; Watson, C. The rat brain in stereotaxic coordinates. San Diego: Academic Press; 1982.

Paxinos, G.; Watson, C. The rat brain in stereotaxic coordinates. 3rd ed. San Diego: Academic Press; 1997.

Peterson RP. Magnocellular neurosecretory centers in the rat hypothalamus. J Comp Neurol. 1966; 128:181-190. [PubMed: 4165735]

Pyner S, Coote JH. Identification of branching paraventricular neurons of the hypothalamus that project to the rostroventrolateral medulla and spinal cord. Neuroscience. 2000; 100:549-556. [PubMed: 11098118]

Qin C, Luo M. Neurochemical phenotypes of the afferent and efferent projections of the mouse medial habenula. Neuroscience. 2009; 161:827-837. [PubMed: 19362132]

Rho JH, Swanson LW. A morphometric analysis of functionally defined subpopulations of neurons in the paraventricular nucleus of the rat with observations on the effects of colchicine. J Neurosci. 1989; 9:1375-1388. [PubMed: 2784832]

Rhodes CH, Morrell JI, Pfaff DW. Immunohistochemical analysis of magnocellular elements in rat hypothalamus: distribution and numbers of cells containing neurophysin, oxytocin, and vasopressin. J Comp Neurol. 1981; 198:45-64. [PubMed: 7014660]

Richoux JP, Dubois MP. Neuronal systems immunologically related to the somatostatin system in the garden dormouse. Cell Tissue Res. 1980; 209:455-472. [PubMed: 6105921]

Risold PY, Swanson LW. Connections of the rat lateral septal complex. Brain Res Brain Res Rev. 1997; 24:115-95. [PubMed: 9385454]

Saper CB, Sawchenko PE. Magic peptides, magic antibodies: guidelines for appropriate controls for immunohistochemistry. J Comp Neurol. 2003; 465:161-163. [PubMed: 12949777]

Saper CB, Loewy AD, Swanson LW, Cowan WM. Direct hypothalamo-autonomic connections. Brain Res. 1976; 117:305-312. [PubMed: 62600]

Sawchenko PE, Swanson LW. Immunohistochemical identification of neurons in the paraventricular nucleus of the hypothalamus that project to the medulla or to the spinal cord in the rat. J Comp Neurol. 1982; 205:260-272. [PubMed: 6122696]

Sawchenko PE, Swanson LW. Localization, colocalization, and plasticity of corticotropin-releasing factor immunoreactivity in rat brain. Fed Proc. 1985; 44:221-227. [PubMed: 2981743]

Sawchenko PE, Swanson LW, Vale WW. Coexpression of corticotropin-releasing factor and vasopressin immunoreactivity in parvocellular neurosecretory neurons of the adrenalectomized rat. Proc Natl Acad Sci U S A. 1984a; 81:1883-1887. [PubMed: 6369332]

Sawchenko PE, Swanson LW, Vale WW. Corticotropin-releasing factor: coexpression within distinct subsets of oxytocin-, vasopressin-, and neurotensin-immunoreactive neurons in the hypothalamus of the male rat. J Neurosci. 1984b; 4:1118-1129. [PubMed: 6609226]

Shimada O, Ishikawa H. Somatostatin-containing neurons in the mouse brain: an immunohistochemical study and comparison with the rat brain. Arch Histol Cytol. 1989; 52:201212. [PubMed: 2570600]

Sidman, RL. Atlas of the mouse brain and spinal cord. Boston: Harvard University Press; 1971.

Simmons DM, Swanson LW. High-resolution paraventricular nucleus serial section model constructed within a traditional rat brain atlas. Neurosci Lett. 2008; 438:85-89. [PubMed: 18479821]

Simmons DM, Swanson LW. Comparison of the spatial distribution of seven types of neuroendocrine neurons in the rat paraventricular nucleus: toward a global 3D model. J Comp Neurol. 2009; 516:423-441. [PubMed: 19655400] 
Sofroniew MV, Weindl A, Schrell U, Wetzstein R. Immunohistochemistry of vasopressin, oxytocin and neurophysin in the hypothalamus and extrahypothalamic regions of the human and primate brain. Acta Histochem Suppl. 1981; 24:79-95. [PubMed: 6785843]

Stocker SD, Simmons JR, Stornetta RL, Toney GM, Guyenet PG. Water deprivation activates a glutamatergic projection from the hypothalamic paraventricular nucleus to the rostral ventrolateral medulla. J Comp Neurol. 2006; 494:673-685. [PubMed: 16374796]

Swanson LW. Immunohistochemical evidence for a neurophysin-containing autonomic pathway arising in the paraventricular nucleus of the hypothalamus. Brain Res. 1977; 128:346-353. [PubMed: 301423]

Swanson, LW. Organization of the mammalian neuroendocrine system. In: Bloom, FE., editor. Handbook of physiology, the nervous system. Vol. IV. Baltimore: Waverly Press; 1986. p. 169-190.

Swanson, LW. The hypothalamus. In: Björklund, A.; HöTkfelt, T.; Swanson, LW., editors. Handbook of chemical neuroanatomy, integrated systems of the CNS, part I. Amsterdam: Elsevier; 1987. p. 1-124.

Swanson, LW. Brain maps: structure of the rat brain. Amesterdam: Elsevier; 2004.

Swanson LW, Kuypers HG. The paraventricular nucleus of the hypothalamus: cytoarchitectonic subdivisions and organization of projections to the pituitary, dorsal vagal complex, and spinal cord as demonstrated by retrograde fluorescence double-labeling methods. J Comp Neurol. 1980; 194:555-570. [PubMed: 7451682]

Swanson LW, McKellar S. The distribution of oxytocin-and neurophysin-stained fibers in the spinal cord of the rat and monkey. J Comp Neurol. 1979; 188:87-106. [PubMed: 115910]

Swanson LW, Sawchenko PE. Paraventricular nucleus: a site for the integration of neuroendocrine and autonomic mechanisms. Neuroendocrinology. 1980; 31:410-417. [PubMed: 6109264]

Swanson LW, Sawchenko PE. Hypothalamic integration: organization of the paraventricular and supraoptic nuclei. Annu Rev Neurosci. 1983; 6:269-324. [PubMed: 6132586]

Swanson LW, Sawchenko PE, Rivier J, Vale WW. Organization of ovine corticotropin-releasing factor immunoreactive cells and fibers in the rat brain: an immunohistochemical study. Neuroendocrinology. 1983; 36:165-186. [PubMed: 6601247]

Tramu G, Croix C, Pillez A. Ability of the CRF immunoreactive neurons of the paraventricular nucleus to produce a vasopressin-like material. Immunohistochemical demonstration in adrenalectomized guinea pigs and rats. Neuroendocrinology. 1983; 37:467-469. [PubMed: 6606790]

Tuli JS, Smith JA, Morton DB. Effects of acute and chronic restraint on the adrenal gland weight and serum corticosterone concentration of mice and their faecal output of oocysts after infection with Eimeria apionodes . Res Vet Sci. 1995; 59:82-86. [PubMed: 8525092]

van den, Pol AN. The magnocellular and parvocellular paraventricular nucleus of rat: intrinsic organization. J Comp Neurol. 1982; 206:317-345. [PubMed: 7096631]

Viau V. Functional cross-talk between the hypothalamic-pituitary-gonadal and -adrenal axes. J Neuroendocrinol. 2002; 14:506-513. [PubMed: 12047726]

Wang Q, Burkhalter A. Area map of mouse visual cortex. J Comp Neurol. 2007; 502:339-357. [PubMed: 17366604]

Watts AG. Glucocorticoid regulation of peptide genes in neuroendocrine CRH neurons: a complexity beyond negative feedback. Front Neuroendocrinol. 2005; 26:109-130. [PubMed: 16289311]

Wickersham IR, Lyon DC, Barnard RJ, Mori T, Finke S, Conzelmann KK, Young JA, Callaway EM. Monosynaptic restriction of transsynaptic tracing from single, genetically targeted neurons. Neuron. 2007; 53:639-647. [PubMed: 17329205]

Young EA, Akana S, Dallman MF. Decreased sensitivity to glucocorticoid fast feedback in chronically stressed rats. Neuroendocrinology. 1990a; 51:536-542. [PubMed: 2162011]

Young WS, Reynolds K, Shepard EA, Gainer H, Castel M. Cell-specific expression of the rat oxytocin gene in transgenic mice. J Neuroendocrinol. 1990b; 2:917-925. [PubMed: 19215438] 

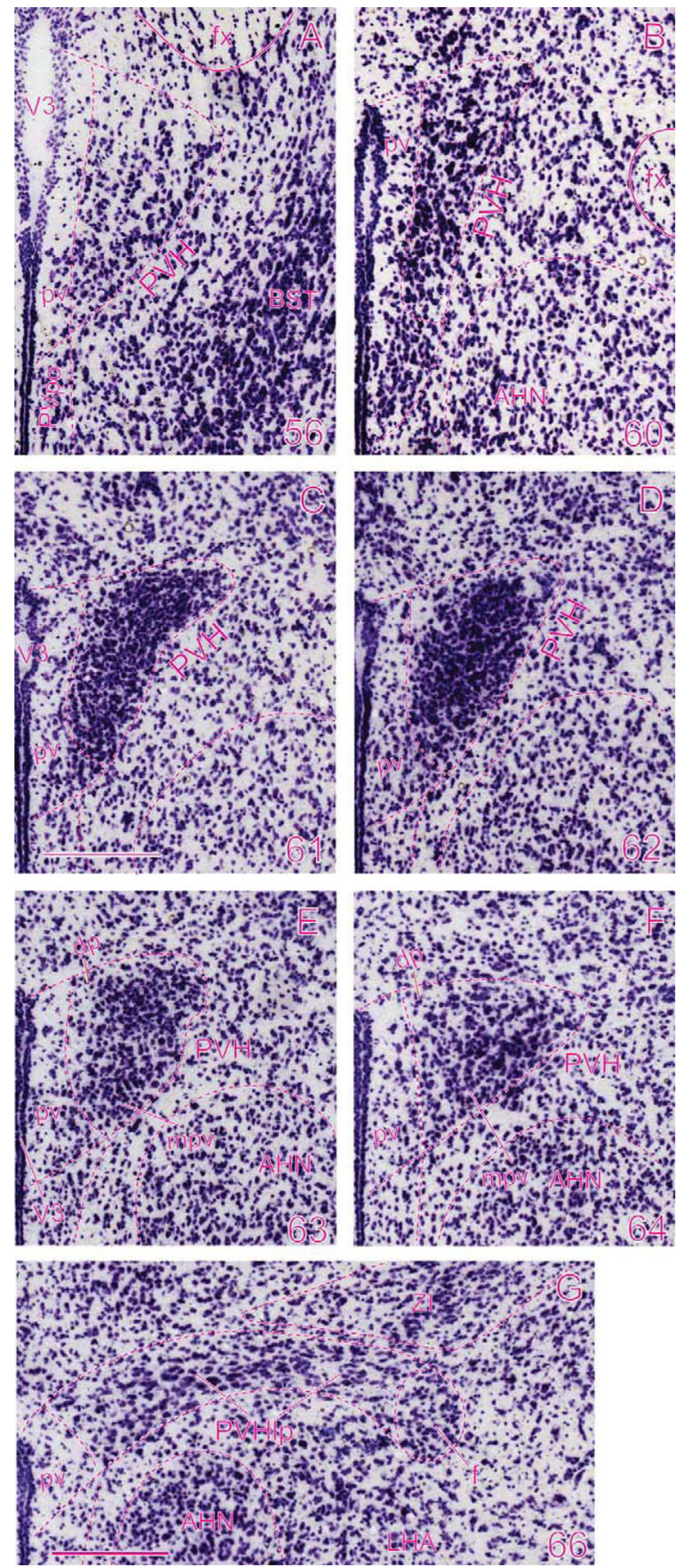

Figure 1.

Nissl-stained digital images of seven representative levels through the PVH of the C57BL/6J mouse. A represents level 56, B-F levels 60-64, and G level 66 of the Allen Reference Atlas (ARA; Dong, 2007). Scale bars $=200 \mu \mathrm{m}$. 

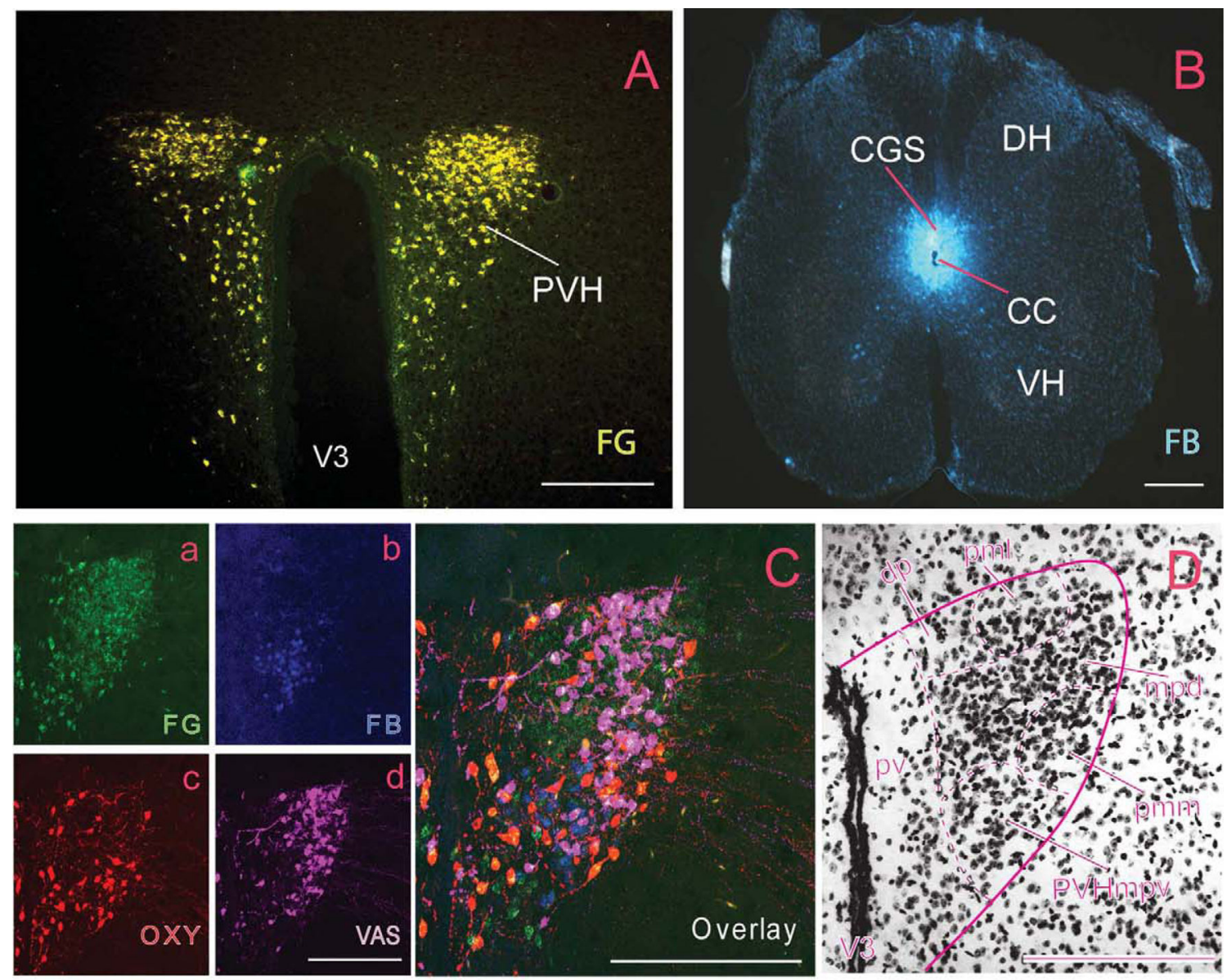

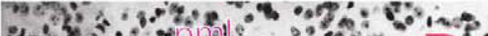
(8) $30.0 \%$

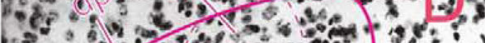

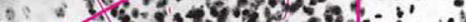

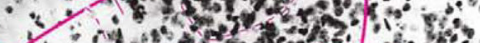
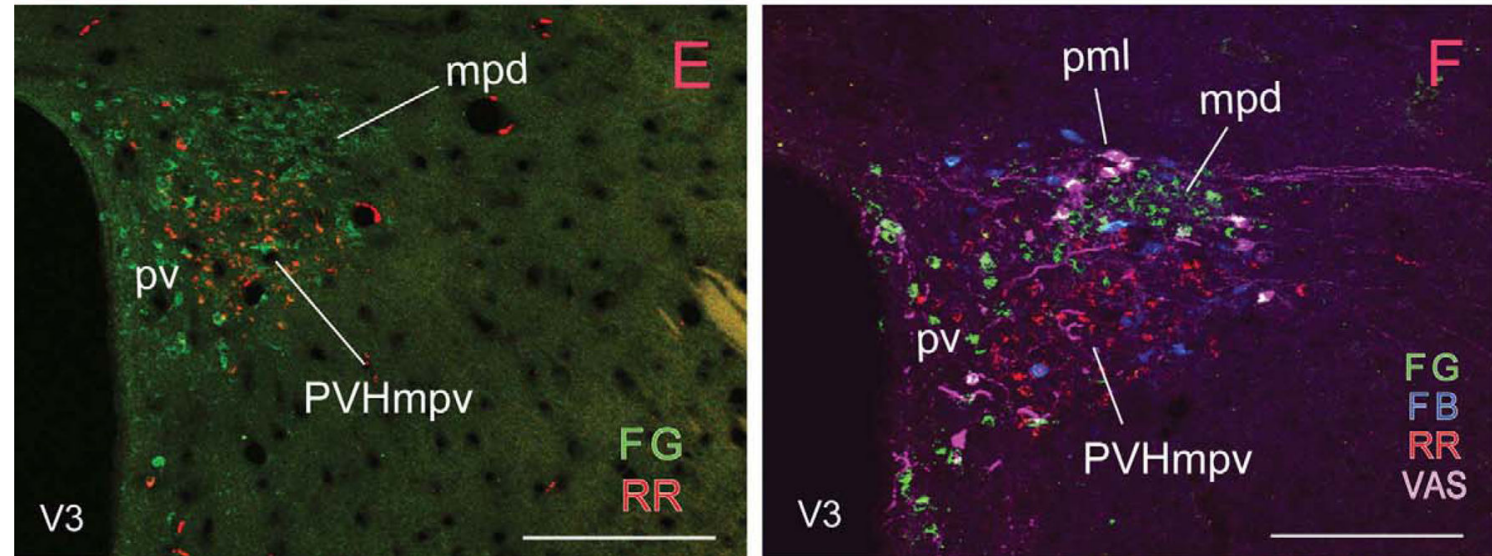

Figure 2.

Photomicrographs illustrating our general strategy for characterizing the cyto- and chemoarchitecture of the mouse PVH. A shows a representative photomicrograph of the mouse PVH with fluorogold (FG)-labeled hypothalamic neuroendocrine neurons after tail vein injection of the retrograde tracer. These animals also received stereotaxic injections of the retrograde tracers fast blue (FB) and/or Red Retrobeads (RR) into the spinal cord and/or the DMX, respectively to label the descending preautonomic neurons in the PVH (PVHd). B illustrates a representative injection site of FB restricted to the CGS. Brain sections from 
these animals were used to perform multiple immunostainings with different combinations to reveal distributions of all neuroendocrine hormonal peptides (OXY, VAS, CRH, SS, and TRH) in the PVH. C shows a multifluorescent, confocal photomicrograph of FG-labeled neuroendocrine neurons (green), FB-labeled CGS-projecting neurons (blue), and OXY (red)- and VAS (magenta)-immunopositive neurons. Note how neurons of these two magnocellular neuroendocrine populations are double labeled with FG, whereas singly labeled FG neurons are presumably parvicellular neuroendocrine neurons. Each neuronal phenotype is displayed separately as shown in a-d. Topological spatial distributions of these different neuronal populations were compared with Nissl-stained cytoarchitecture of the $\mathrm{PVH}$ in adjacent histological sections illustrated in $\mathrm{D}$, which allows more precise delineation of different parts of the PVH. E shows a representative level of the PVH containing FGlabeled neuroendocrine neurons (green) and RR-labeled DMX-projecting preautonomic neurons (red). F shows a representative confocal photomicrograph, which simultaneously reveals the entire population of hypothalamic neuroendocrine neurons labeled with FG (green), VAS-immunopositive neurons (magenta), descending IML-projecting neurons labeled with FB (blue), and descending DMX-projecting neurons labeled with RR (red). Scale bars $=200 \mathrm{~lm}$. 

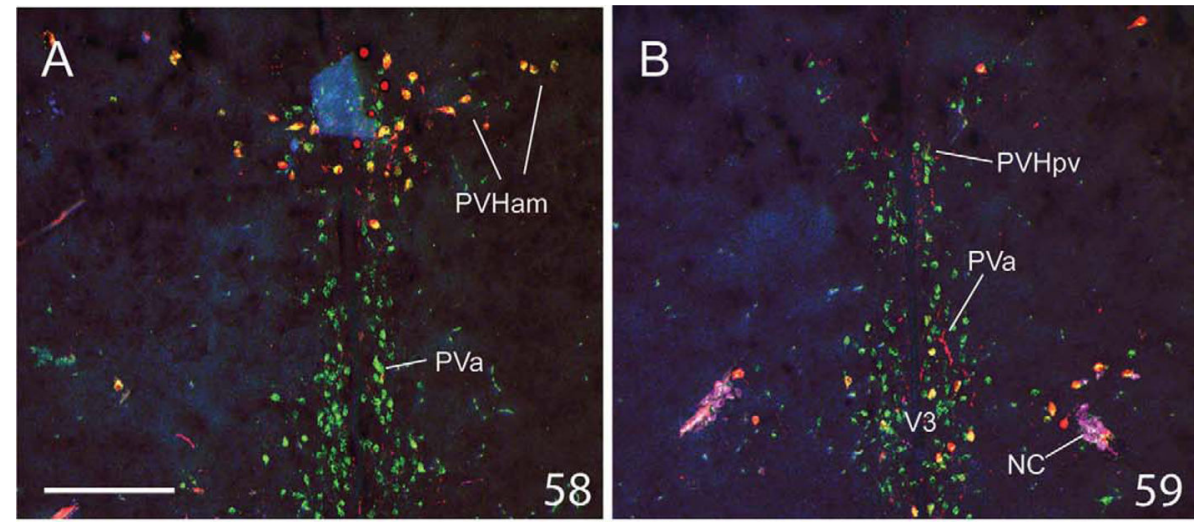

Key:

- FG
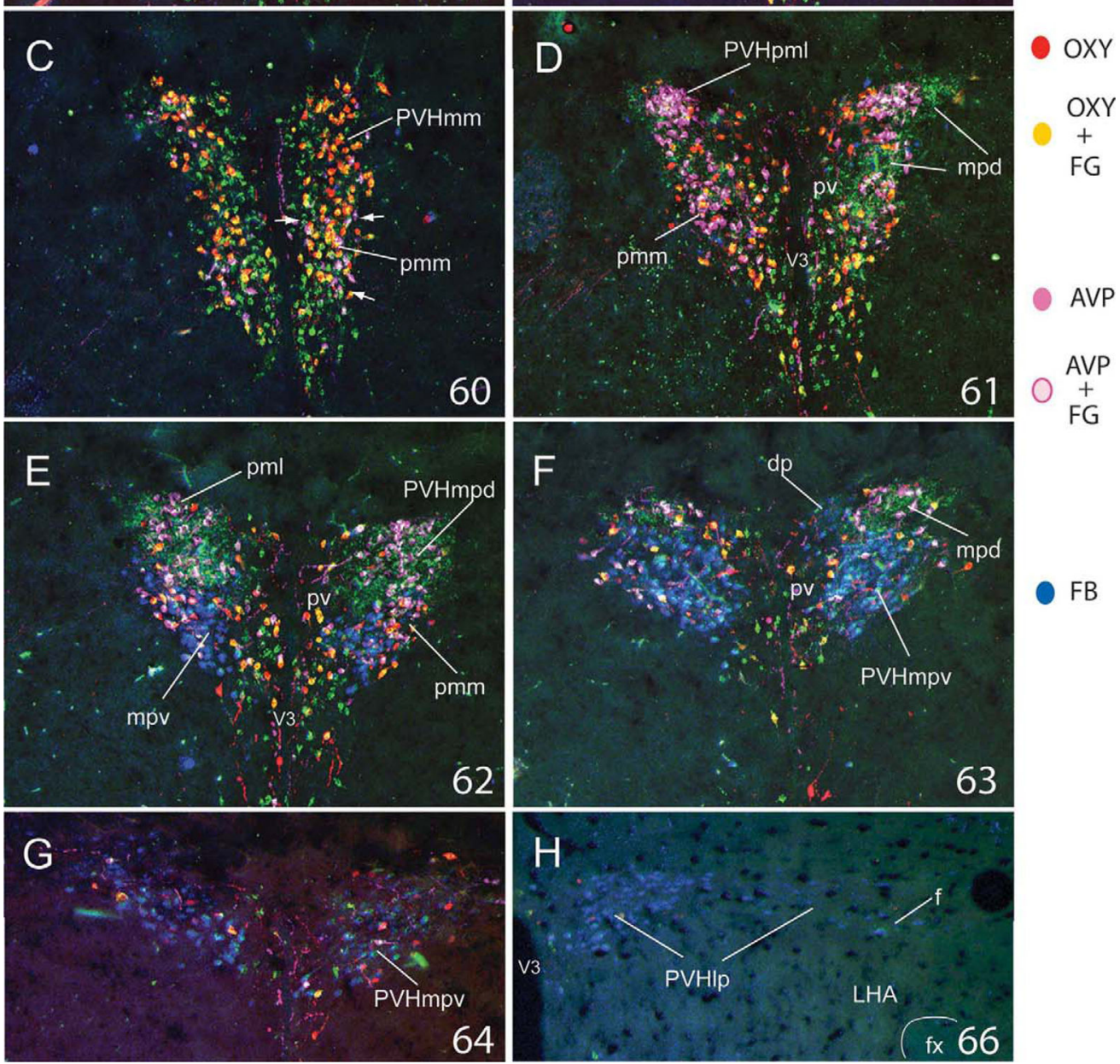

Figure 3.

Confocal photomicrographs show a series of coronal sections (one in four series, $25 \mathrm{~lm}$ thickness) across eight levels of the PVH (experimental case T10; noncolchicine treated) in which four neuronal populations are labeled with different fluorescent markers. The parvicellular neuroendocrine neurons are singly labeled with the retrograde marker FG (green). Two magnocellular neuroendocrine populations are double labeled with FG (green) and OXY (red) or VAS (magenta), whereas CGS-projecting descending neurons are labeled with FB (blue; for injection site see Fig. 2B). Scale bar $=200 \mathrm{~lm}$. 

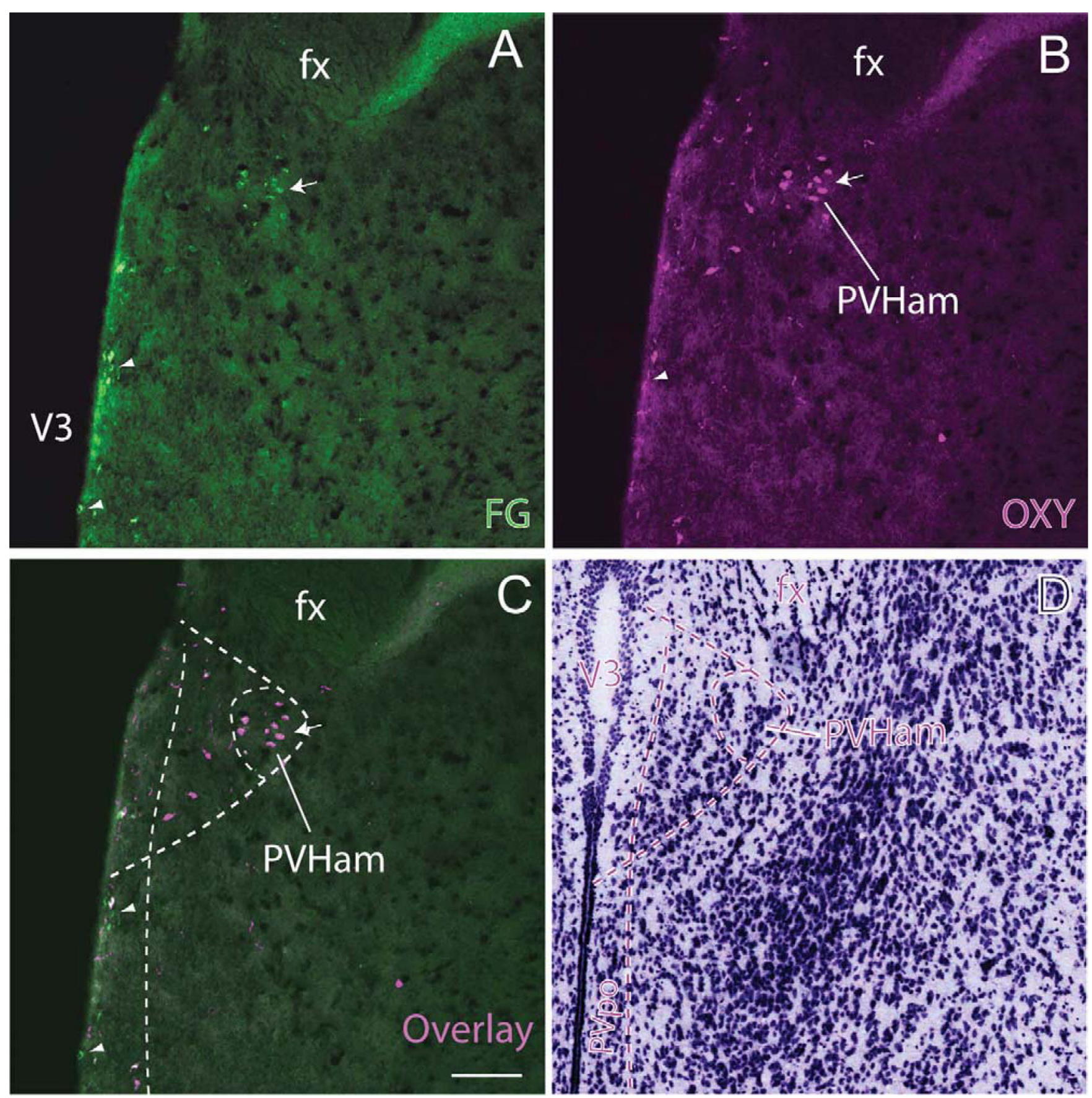

Figure 4.

Confocal photomicrographs showing OXY-immunoreactive neurons in the PVHam double labeled with FG (A-C). Note that the vicinity of the PVHam contains very few singly labeled FG neurons (e.g. white arrow in A), which are presumably parvicellular neuroendocrine cells. $\mathbf{D}$ is a digital image of a Nissl-stained section from the ARA (level 56; see Fig. 1A) that represents the corresponding cytoarchitectural reference for the PVHam. Scale bar $=200 \mu \mathrm{m}$. 

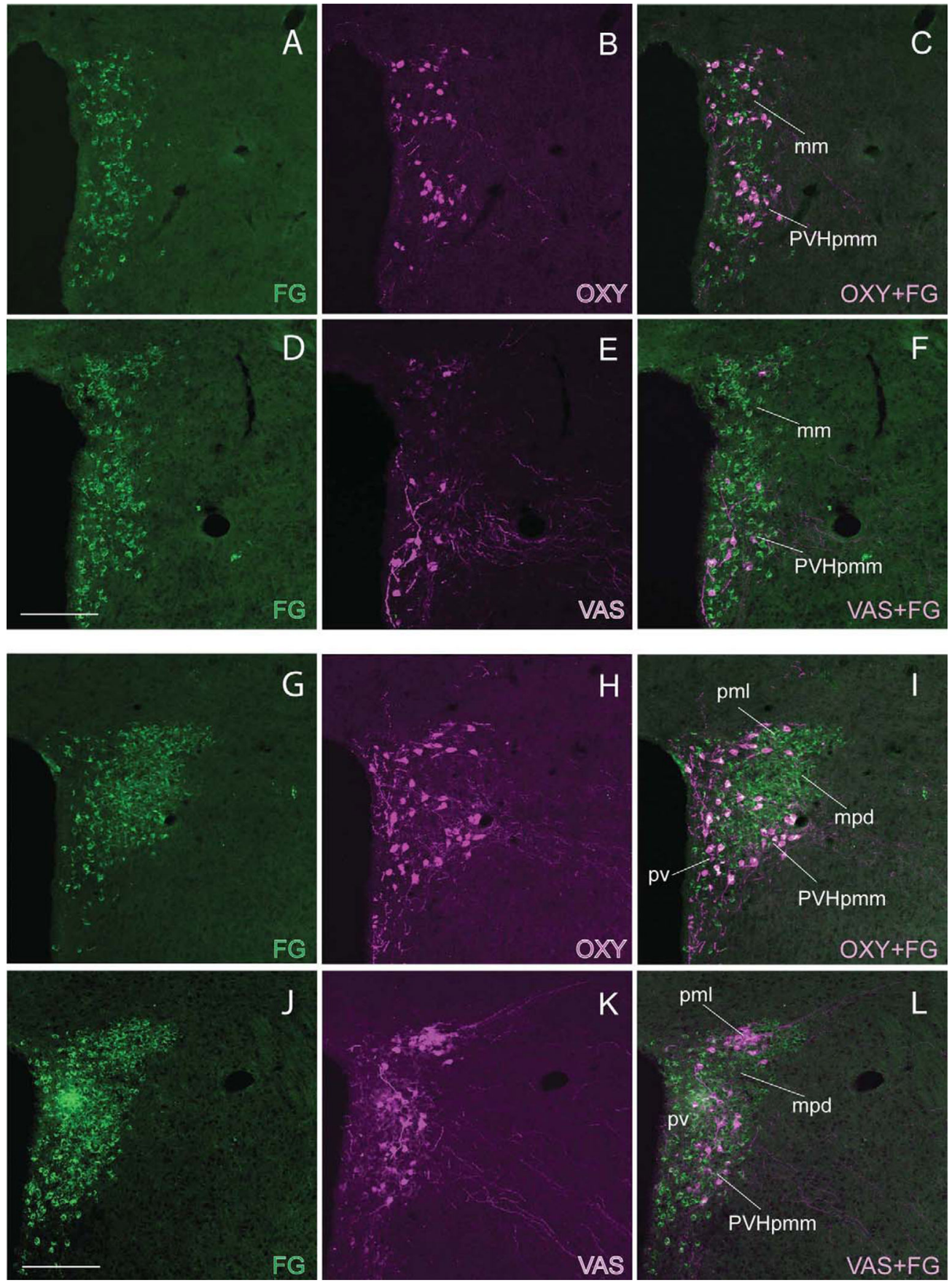

Figure 5.

Distributions of two magnocellular neuroendocrine populations, OXY (A-C,G-I) and VAS (D-F,J-L), are compared in two different coronal levels of the PVH (from two different brains of noncolchicine-pretreated mice). The whole population of neuroendocrine neurons is labeled with FG via tail vein injection (as shown in A,D,G,J), whereas OXY (B,H) or VAS (E,K) neurons were immunostained. Note that almost all OXY (C,I)- or VAS (F,L)immnopositive neurons are neuroendocrine neurons as indicated by their colocalization with FG. At the rostral level (A-F), OXY neurons are distributed in both the PVHmm and the 
PVHpmm, whereas VAS neurons are located primarily in the PVHpmm. At the caudal level, VAS neurons form a densely packed "ball" in the PVHpml (K,L), and OXY neurons are distributed more medially in the PVHpv (H,I). At these two levels, the PVHpmm contains both OXY and VAS neurons (I,L). Scale bars $(\mathrm{D}, \mathrm{J})=200 \mu \mathrm{m}$. 

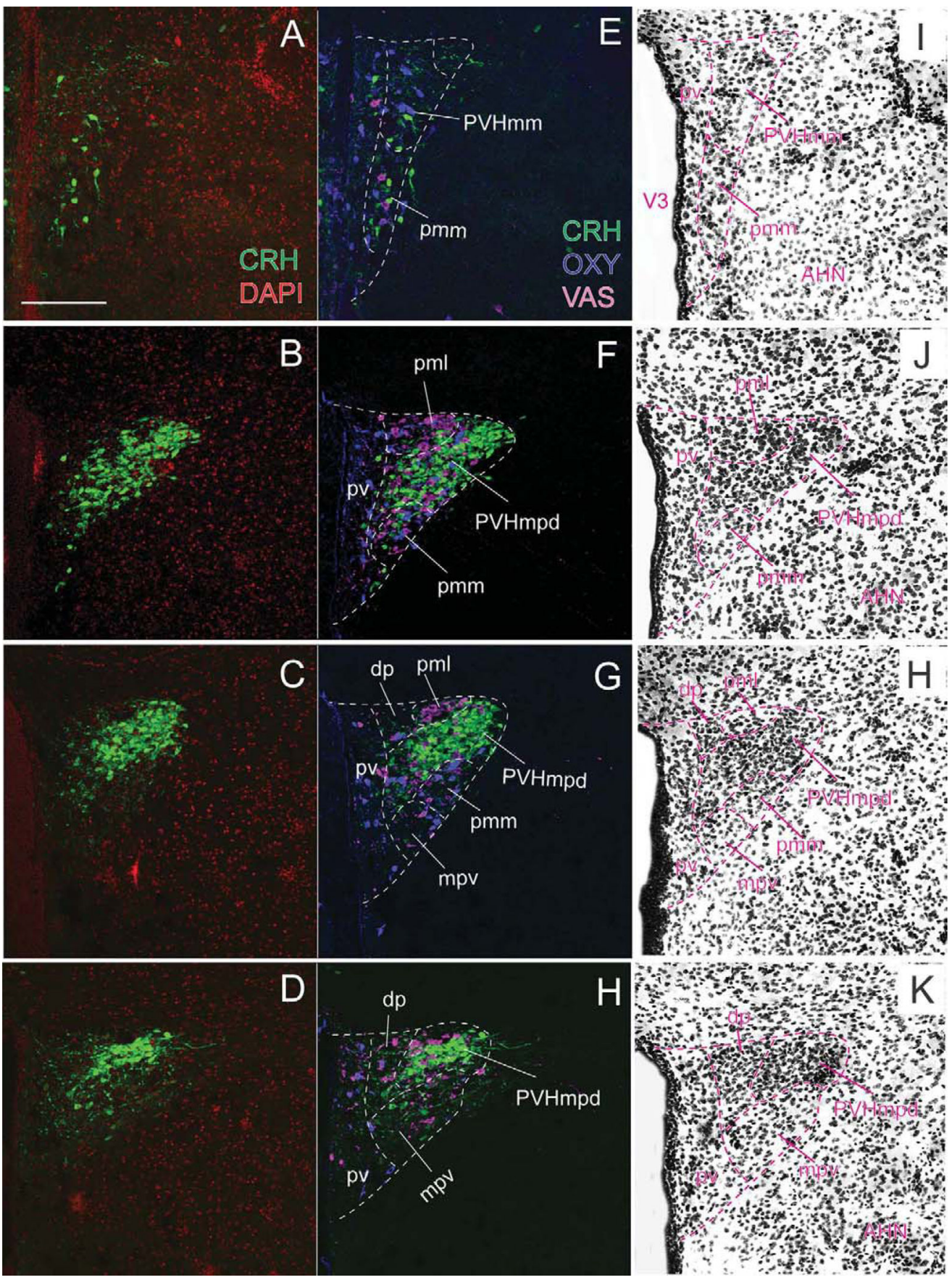

Figure 6.

Confocal photomicrographs showing the distribution of CRH neurons (A-D, with nuclear counterstain DAPI) and their topological spatial relation with OXY and VAS neurons (E-H) in four coronal levels across the PVH (one in four series, $25 \mathrm{~lm}$ thickness). Adjacent thioninstained sections are shown for cytoarchitectural reference (I-K). Although no single neuronal phenotype is restricted to a single subdivision, the same types of neurons do tend to cluster together (i.e., CRH neurons are concentrated in the PVHmpd; the PVHpml contains 
primarily VAS neurons; the PVHpmm contains both OXY and VAS neurons), allowing further delineation of the PVH. Scale bar $=200 \mu \mathrm{m}$. 

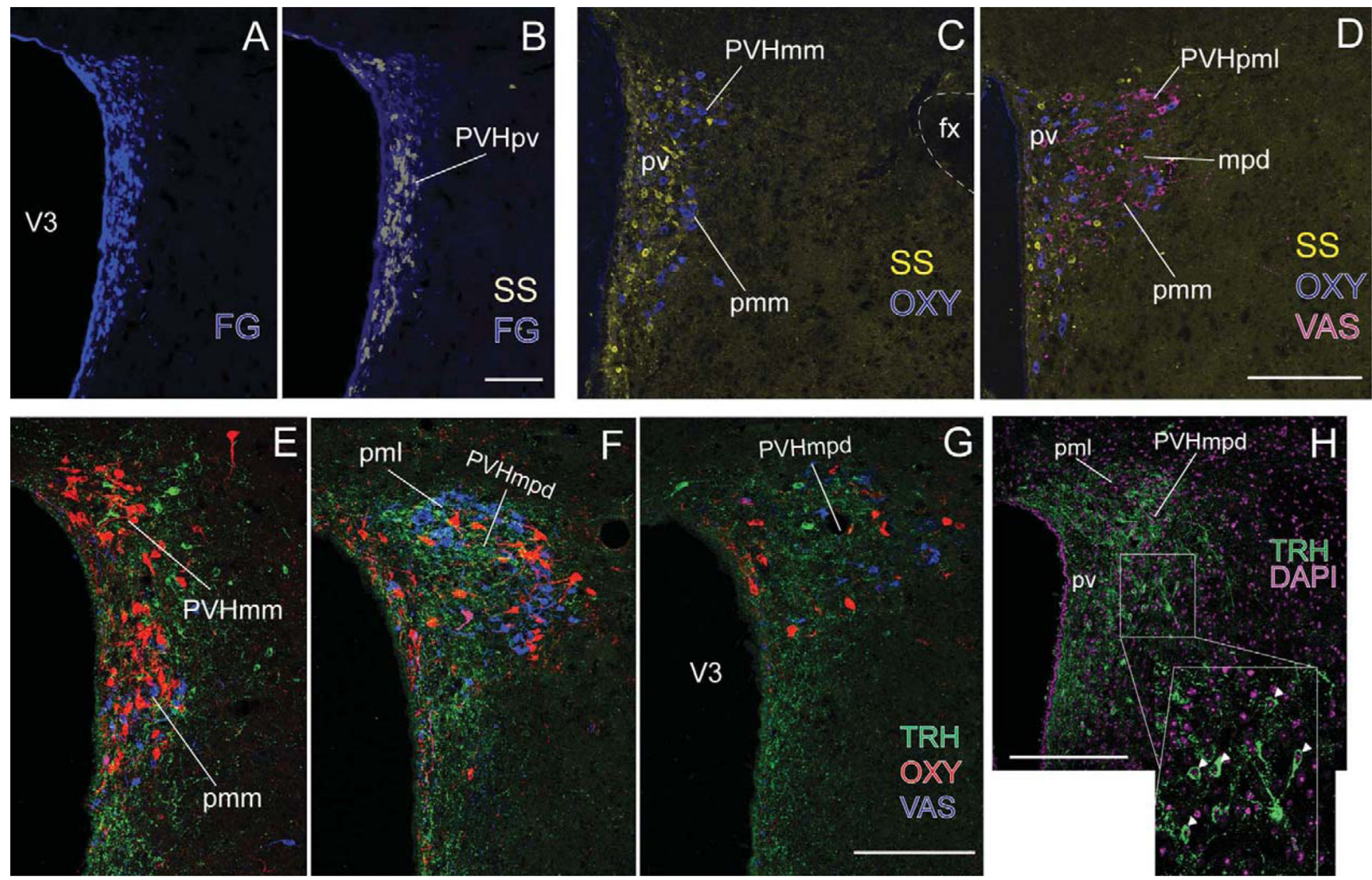

Figure 7.

$\mathbf{A}$ and $\mathbf{B}$ show the distribution of SS-immunoreative neurons in the PVHpv and periventricular nucleus, compared with the distribution of FG-labeled neuroendocrine neurons. $\mathbf{C}$ and $\mathbf{D}$ show the spatial correlations of SS (yellow) neurons with OXY (blue in C,D) and VAS (magenta in D) in the PVH. Most SS neurons are medial to the OXY neurons in the PVHpv. E-G show the spatial distribution of TRH-immunopositive neurons (green) in the PVH and their spatial correlation with OXY (red) and VAS (blue) in the PVH. Most of the TRH neurons are distributed in the PVHmpd as shown in F,H. In H, nuclei were revealed with DAPI, to facilitate identification of individual TRH neurons (as shown in the boxed area). All brain sections shown were obtained from mice that had received colchicine injections. Scale bars $=200 \mu \mathrm{m}$. 

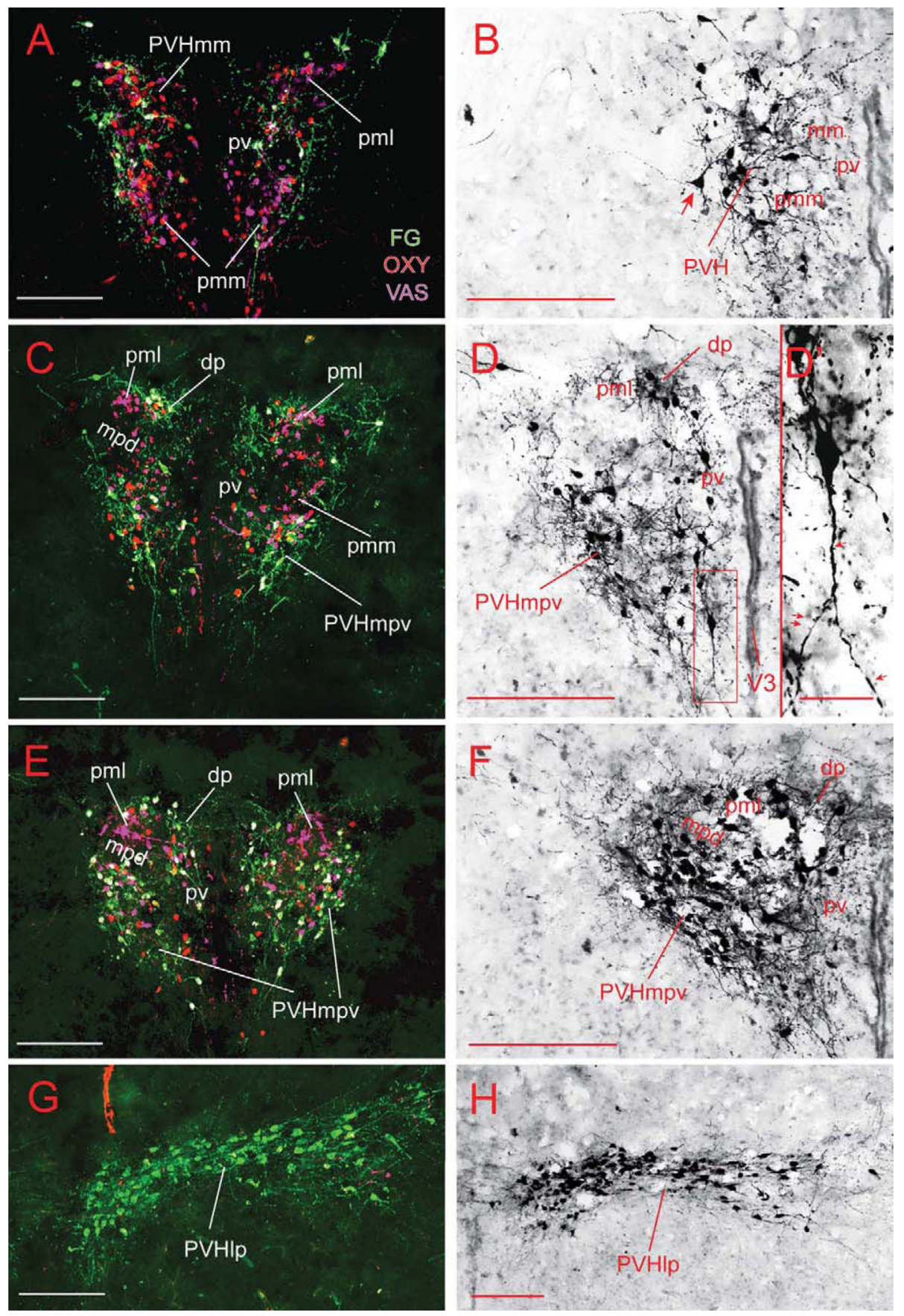

Figure 8.

Photomicrographs show the distribution of FG-labeled IML-projecting neurons in the PVH and their spatial correlation with OXY and VAS neurons (A,C,E,G; experimental case T11; noncolchicine pretreated). In the corresponding adjacent sections, Golgi-like neuronal morphology of the FG-labeled descending neurons is revealed with an immunohistochemical method (B,D,F,H). D' is a magnified enlarged image of a FG-labeled descending neuron in $\mathrm{D}$ (within the red box), where arrows indicate dendritic spines. Scale bars $=200 \mu \mathrm{m}$. 

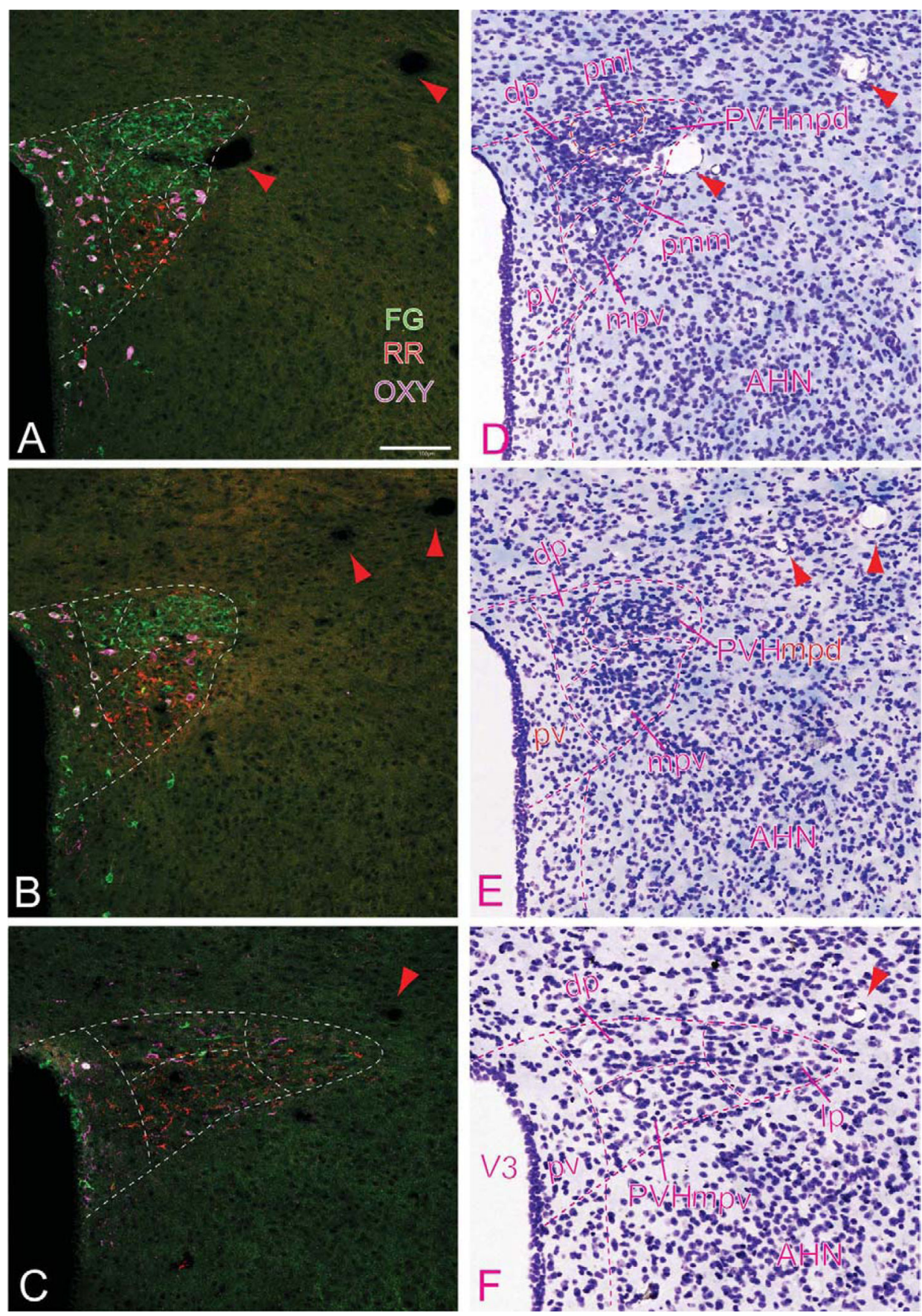

Figure 9.

Photomicrographs show the distribution of Red Retrobeads (RR)-labeled DMX-descending projection neurons and their spatial correlation with FG-labeled neuroendocrine neurons (green) and immunostained OXY neurons (magenta) in three coronal levels of the mouse PVH (A-C). The adjacent Nissl-stained sections show their corresponding cytoarchitectural references (D-F). The red arrowheads point to the same blood vessels in each of the corresponding levels. Please note that the most RR-labeled neurons are distributed in the 
PVHmpv and its adjacent PVHlp. These photomicrographs are from experimental case SCDMIV2, which was not pretreated with colchicine. Scale bar $=100 \mu \mathrm{m}$. 

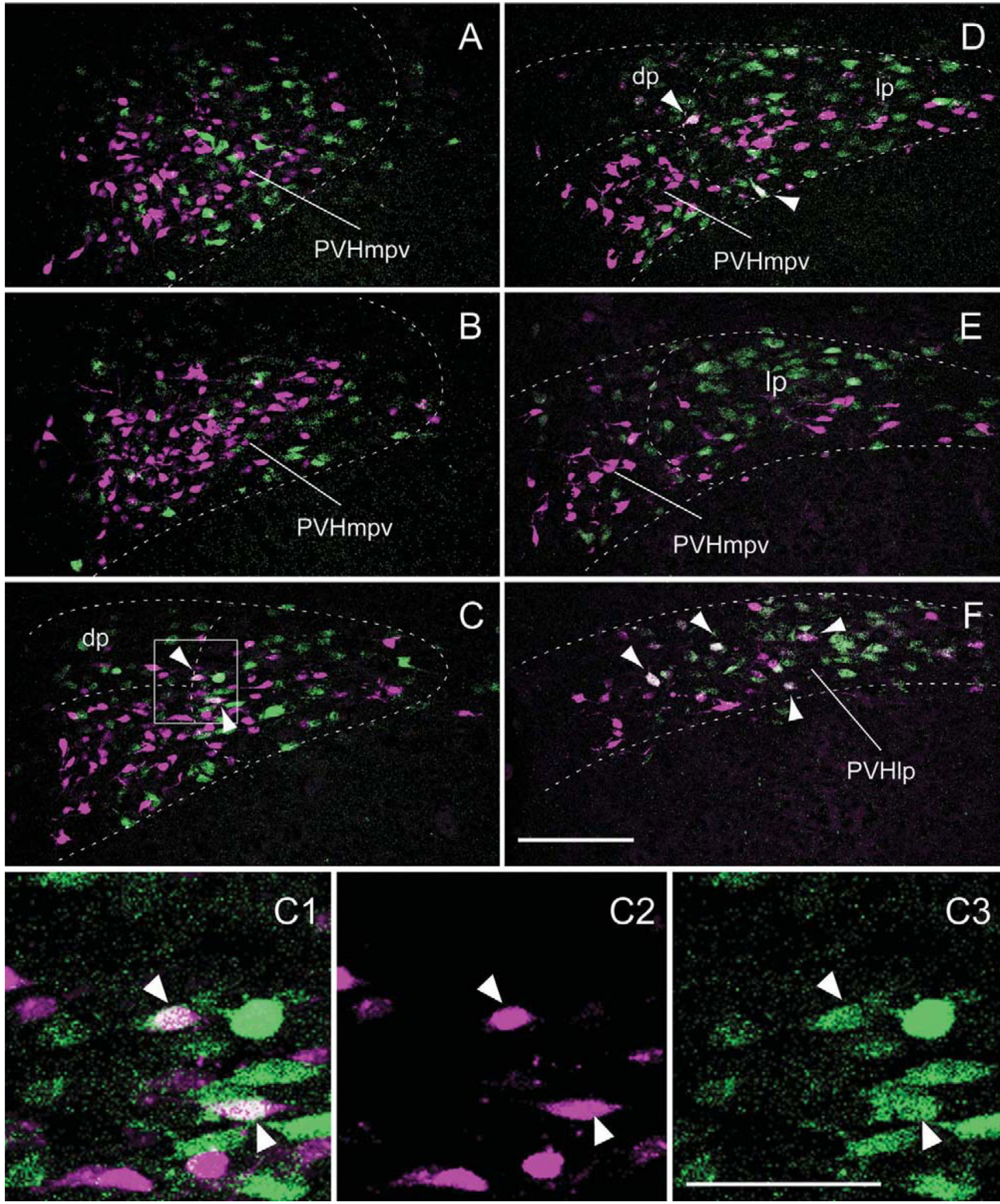

Figure 10.

Confocal photomicrographs show spatial distributions of two populations of descending preautonomic neurons that project to the IML (green, FG-labeled neurons) and/or to the DMX (megenta, FB-labeled neurons) at six contiguous coronal levels through the PVH (25 $\mu \mathrm{m}$ thickness; A-F). Arrowheads in A-F indicate double-labeled neurons (white) that presumably send collateral projections to both the IML and DMX. C1-C3 are enlarged images of the boxed area in $\mathrm{C}$ to show neurons single labeled with FB (C2) or FG (C3) and double labeled with both tracers $(\mathrm{C} 1)$. The mouse used in this experiment did not receive a 
cholchicine injection. A-F are at the same magnification. Scale bars $=50 \mu \mathrm{m}$ in $\mathrm{C} 3 ; 100 \mu \mathrm{m}$ in F. 


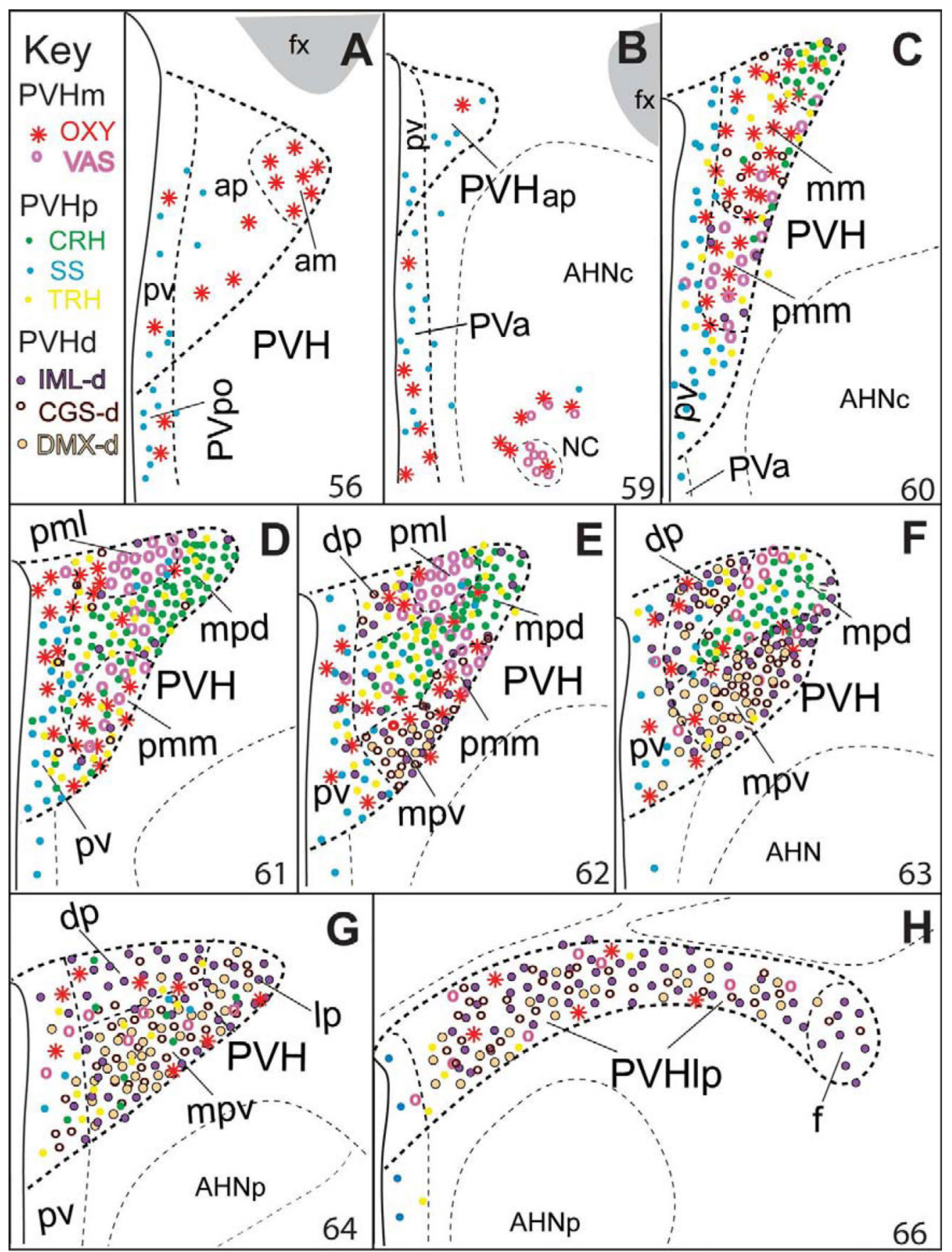

Figure 11.

Schematic drawings illustrating the delineations of the PVH in the mouse brain, which are determined based on distributions of eight neuronal phenotypes in two major neuroendocrine divisions (PVHm, including OXY and VAS; and PVHp, including CRH, $\mathrm{SS}$, and $\mathrm{TRH}$ ) and three descending preautonomic populations (PVHd) that project to the IML (IML-d), to the CGS (CGS-d), and to the DMX (DMX-d). Schematic drawings of the PVH, based on the present data, were modified from eight coronal levels (levels 57, 59, 6064, and 66; see Fig. 1 for cytoarchitectural reference) of the ARA (Dong, 2007). 


\section{The orgnization of the $\mathrm{PVH}$ in mice}

\section{Anterior (level 56-62) \\ Posterior (63-66)}

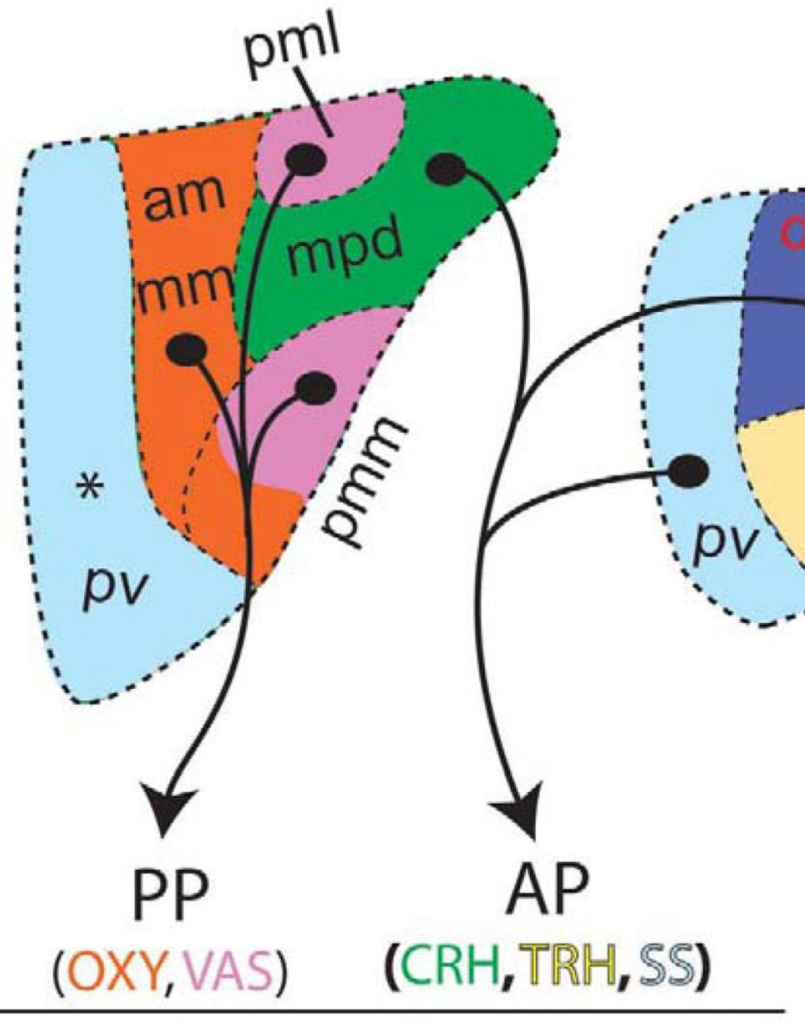

\section{Neuroendocrine}

Figure 12.

Schematic model of the PVH cyto- and chemoarchitecture in the mouse. In general, the anterior (rostral) two-thirds of the PVH (ARA levels 56-62) contains the vast majority of magnocellular neuroendocrine neurons (PVHm) that project to the posterior pituitary (PP) and parvicellular neuroendocrine neurons (PVHp) that project to the median eminence (ME). The posterior (caudal) one-third of the PVH (ARA levels 63-66) contains most of the descending preautonomic neurons. *indicates the anterior PVHpv, along with the posterior PVHpv, also sends projections to the AP. The line was not drawn here to avoid confusion. 


\section{TABLE 1}

\section{Primary Antibodies Used in This Study}

\begin{tabular}{lll}
\hline Target & Antigen & Source \\
\hline Vasopressin & $\begin{array}{l}\text { Synthetic Arg8 vasopressin (antigen } \\
\text { sequence: H-Cys-Tyr-Phe-Gln-Asn-Cys-Pro- } \\
\text { Arg-Gly-NH2) }\end{array}$ & $\begin{array}{l}\text { Peninsula Laboratories; guinea pigs } \\
\text { polyclonal; T-5048 }\end{array}$ \\
& $\begin{array}{l}\text { Oxytocin conjugated to thyroglobulin } \\
\text { Oxytocin }\end{array}$ & $\begin{array}{l}\text { Millipore; mouse monoclonal; } \\
\text { MAB5296 }\end{array}$ \\
Thyrotropin-releasing hormone & $\begin{array}{l}\text { Synthetic pre-pro-TRH160-169 conjugated } \\
\text { to bovine thyroglobulin }\end{array}$ & $\begin{array}{l}\text { M. Wessendorf (University of } \\
\text { Minnesotal); rabbit polyclonal; appT }\end{array}$ \\
& $\begin{array}{l}\text { Somatostatin conjugated to BSA } \\
\text { Somatostatin }\end{array}$ & $\begin{array}{l}\text { Millipore; rabbit polyclonal; AB5494 } \\
\text { Somatostatin }\end{array}$ \\
& $\begin{array}{l}\text { Synthetic somatostatin15-28 conjugated to } \\
\text { keyhole impet hemocyanin }\end{array}$ & $\begin{array}{l}\text { ImmunoStar (Hudson, WI); rabbit } \\
\text { polyclonal; 20067 }\end{array}$ \\
Corticotropin-releasing hormone & $\begin{array}{l}\text { Synthetic rat corticotropin-releasing } \\
\text { hormone conjugated to human a-globulin }\end{array}$ & W. Vale (Salk Institute, La Jolla, \\
& CA); rabbit polyclonal; 70
\end{tabular}


TABLE 2

Secondary Antibodies Used in This Study

\begin{tabular}{|l|c|c|c|}
\hline Secondary antibodies & Source & Catalog Nos. & Working dilution \\
\hline Goat biotinylated anti-rabbit & Vector & BA-1000 & $1: 200$ \\
\hline Goat biotinylated anti-mouse & Vector & BA-9200 & $1: 200$ \\
\hline Goat biotinylated anti-guinea pig & Vector & BA-7000 & $1: 200$ \\
\hline Donkey anti-mouse cy3 & Jackson & $715-165-151$ & $1: 500$ \\
\hline Donkey anti-rabbit FITC & Jackson & $711-095-152$ & $1: 200$ \\
\hline Biotin-SP-conjugated donkey anti-rabbit & Jackson & $711-065-152$ & $1: 200$ \\
\hline Biotin-SP-conjugated donkey anti-rabbit & Jackson & $711-065-152$ & $1: 500$ \\
\hline Biotin-SP-conjugated donkey anti-rabbit & Jackson & $711-065-152$ & $1: 500$ \\
\hline Cy5-conjugated streptavidin & Jackson & $016-170-084$ & $1: 500$ \\
\hline Streptavidin Alexa Fluor 488 & Invitrogen & S32354 & $1: 500$ \\
\hline Donkey anti-rabbit IgG (HpL) Alexa Fluor 488 & Invitrogen & A-21206 & $1: 500$ \\
\hline Donkey anti-mouse IgG (HpL) Alexa Fluor 568 & Invitrogen & A-10037 & $1: 500$ \\
\hline Goat anti-mouse IgG (H L) Alexa Fluor 546 & Invitrogen & A-11003 & $1: 500$ \\
\hline Goat anti-rabbit IgG (HpL) Alexa Fluor 488 & Invitrogen & A-11008 & $1: 500$ \\
\hline Goat anti-guinea pig Alexa Fluro 647 & Invitrogen & A-21450 & $1: 500$ \\
\hline
\end{tabular}

